Supporting information for:

\title{
Highly Enantioselective Insertion of Carbenoids into O-H Bonds of Phenols: An Efficient Approach to Chiral $\alpha$-Aryloxycarboxylic Esters
}

\author{
Chao Chen, ${ }^{\mathrm{b}}$ Shou-Fei Zhu, ${ }^{\mathrm{a}}$ Bin Liu, ${ }^{\mathrm{a}} \mathrm{Li}-$ Xin Wang, ${ }^{\text {a }}$ Qi-Lin Zhou${ }^{*, \mathrm{a}}$ \\ ${ }^{a}$ State Key Laboratory and Institute of Elemento-organic Chemistry, Nankai University, Tianjin 300071, China. \\ ${ }^{\mathrm{b}}$ Institute of Fine Chemicals, East China University of Science and Technology, Shanghai 200237, China
}

\section{CONTENTS:}

1. Typical Procedure for Cu-Catalyzed Asymmetric Insertion of Carbenoid into O-H Bonds of Phenols and Alcohols

2. Analytical Data for $\mathbf{O}-\mathbf{H}$ Insertion Products

3. General Procedure for Hydrolysis of 2-Aryloxypropionates

4. Analytical Data for 2-Aryloxypropanoic Acids

5. NMR Spectra of New $\mathrm{O}-\mathrm{H}$ Insertion Products

6. HPLC and SFC Charts of $\mathrm{O}-\mathrm{H}$ Insertion Products

General. All reactions and manipulations were performed using standard Schlenk techniques. Toluene and n-hexane were distilled from sodium benzophenone ketyl. $\mathrm{CH}_{2} \mathrm{Cl}_{2}, \mathrm{CHCl}_{3}$ and $\mathrm{DME}$ were distilled over $\mathrm{CaH}_{2}$ under nitrogen atmosphere. $\mathrm{CuCl}^{1}{ }^{1} \mathrm{CuPF}_{6}(\mathrm{MeCN})_{4},{ }^{1}$ and $\mathrm{NaBARF}^{2}$ ligands $\mathbf{1},{ }^{3}$ Ph-Binabox, ${ }^{4}$ $(S, S)$-Ph-Box ${ }^{5}$ and $(S, S)-{ }^{i}$ Pr-Pybox ${ }^{6}$ were prepared according to the literatures. Cu(OTf $)_{2}$ was purchased from Aldrich and used directly. NMR spectra were recorded on a Bruker or Varian spectrometer at 400 or $300\left({ }^{1} \mathrm{H}\right.$ NMR), 100 or $75\left({ }^{13} \mathrm{C}\right.$ NMR) $\mathrm{MHz}$ with $\mathrm{CDCl}_{3}$ as solvent. Chemical shifts ( $\delta$ values) were reported in ppm down field from internal $\mathrm{Me}_{4} \mathrm{Si}\left({ }^{1} \mathrm{H}\right.$ and ${ }^{13} \mathrm{C}$ NMR). Optical rotations were determined using a Perkin Elmer 341 MC polarimeter. HRMS were recorded on VG ZAB-HS mass spectrometer with EI resource. Mass spectra were recorded on a LCQ Advantage spectrometer with EI resource. Elemental analyses were performed on Yanaca CDRDER MT-3 instrument. HPLC analyses were performed on a Hewlett Packard Model HP 1100 Series or Waters 2996 chromatography. SFC analyses were performed on Mettler-Toledo Model Analytix SFC.

\section{Typical Procedure for Cu-Catalyzed Asymmetric Insertion of Carbenoid into O-H Bonds of Phenols and Alcohols}

The CuCl (1.9 mg, $0.02 \mathrm{mmol}),\left(S_{a}, S, S\right)-1 \mathrm{a}$ (12.2 mg, $\left.0.024 \mathrm{mmol}\right)$, NaBARF (22.6 mg, $\left.0.024 \mathrm{mmol}\right)$ and $600 \mathrm{mg} 5 \AA \AA$ molecular sieves were introduced into an oven-dried Schlenk tube. $\mathrm{CH}_{2} \mathrm{Cl}_{2}(4 \mathrm{~mL})$ was injected. The solution was stirred at room temperature under the argon atmosphere for $2 \mathrm{~h}$, followed by the additions of phenol (188 mg, $2.0 \mathrm{mmol}$ ) and ethyl $\alpha$-diazopropionate (52 mg, $0.4 \mathrm{mmol}$ ). The resulting

\footnotetext{
${ }^{1}$ Inorg. Synth. Vol. II, p. 1

2 (a) Brookhart, M.; Grant, B.; Volpe, A. F. Organometallics 1992, 11, 3920. (b) Nishida, H.; Takada, N.; Yoshimura, M.; Sonoda, T.; Kobayashi, H. Bull. Chem. Soc. Jpn. 1984, 57, 2600.

${ }^{3}$ Liu, B.; Zhu, S.-F.; Zhang, W.; Chen, C.; Zhou, Q.-L. J. Am. Chem. Soc. 2007, 129, 5834

${ }^{4}$ Uozumi, Y.; Kyota, H.; Kishi, E., Kitayama, K.; Hayashi, T. Tetrahedron: Asymmetry 1996, 7, 1603

${ }^{5}$ Corey, E. J.; Imai, N.; Zhang, H.-Y. J. Am. Chem. Soc. 1991, 113, 728

${ }^{6}$ Nishiyama, H.; Sakaguchi, H.; Nakamura, T.; Horihata, M.; Kondo, M.; Itoh, K. Organometallics 1989, 8, 846.
} 
mixture was stirred at $25{ }^{\circ} \mathrm{C}$ for $3 \mathrm{~h}$. After filtrating and removing solvent in vacuum the product was purified by flash chromatography with ethyl acetate/petroleum ether (1:30). The analytical data for aryloxypropionates are listed below.

\section{Analytical Data for $\mathrm{O}-\mathbf{H}$ Insertion Products}

(R)-(+)-Ethyl 2- phenoxypropionate (4a) ${ }^{7}$

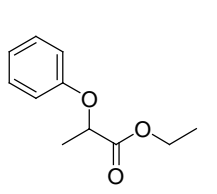

Colorless oil; 87\% yield; ${ }^{1} \mathrm{H}$ NMR: $\delta 7.28$ (t, $\left.J=8.0 \mathrm{~Hz}, 2 \mathrm{H}\right), 6.97$ (t, $\left.J=7.2 \mathrm{~Hz}, 1 \mathrm{H}\right), 6.87$ (d, $J=8.0 \mathrm{~Hz}, 2 \mathrm{H}), 4.75$ (q, $J=6.8 \mathrm{~Hz}, 1 \mathrm{H}), 4.21$ (q, $J=7.2 \mathrm{~Hz}, 2 \mathrm{H}), 1.61$ (d, $J=6.8 \mathrm{~Hz}$, $3 \mathrm{H}), 1.24(\mathrm{t}, J=7.2 \mathrm{~Hz}, 3 \mathrm{H})$; 99\% ee [HPLC condition: Chiralcel OD-H column, $n$-Hexane $/ \mathrm{i}-\mathrm{PrOH}=90: 10$, flow rate $=1.0 \mathrm{~mL} / \mathrm{min}$, wavelength $=210 \mathrm{~nm}, t_{\mathrm{R}}=5.62 \mathrm{~min}$ for $(S)$-enantiomer, $t_{\mathrm{R}}=10.09 \mathrm{~min}$ for $(R)$-enantiomer]; $[\alpha]_{\mathrm{D}}{ }^{18}=+47.2\left(c\right.$ 0.5, MeOH) [lit: $[\alpha]_{\mathrm{D}}{ }^{20}=+47$ (c 2.2, $\mathrm{MeOH})$ for $(R)]^{7}$

\section{(+)-Ethyl 2-(o-tolyloxy)propionate (4b) ${ }^{8}$}

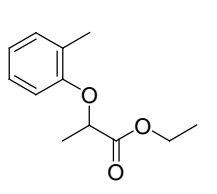

Colorless oil; 71\% yield; ${ }^{1} \mathrm{H}$ NMR: $\delta$ 7.15-7.07 (m, 2H), 6.87 (t, $\left.J=7.2 \mathrm{~Hz}, 1 \mathrm{H}\right), 6.68$ (d, $J$ $=8.4 \mathrm{~Hz}, 1 \mathrm{H}), 4.73(\mathrm{q}, J=6.8 \mathrm{~Hz}, 1 \mathrm{H}), 4.21(\mathrm{q}, J=7.2 \mathrm{~Hz}, 2 \mathrm{H}), 2.28$ (s, 3H), 1.63 (d, $J=$ $7.2 \mathrm{~Hz}, 3 \mathrm{H}$ ), 1.25 (t, $J=6.8 \mathrm{~Hz}, 3 \mathrm{H}$ ); 98\% ee [HPLC condition: Chiralcel OD-H column, $n$-Hexane $/ \mathrm{i}-\mathrm{PrOH}=90: 10$, flow rate $=1.0 \mathrm{~mL} / \mathrm{min}$, wavelength $=210 \mathrm{~nm}, t_{\mathrm{R}}=4.62 \mathrm{~min}$ for minor isomer, $t_{\mathrm{R}}=8.59$ min for major isomer $] ;[\alpha]_{\mathrm{D}}{ }^{18}=+29.5\left(c 0.75, \mathrm{CH}_{2} \mathrm{Cl}_{2}\right)$.

\section{(+)-Ethyl 2-(2-methoxyphenoxy)propionate(4c)}

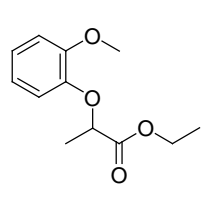

Colorless oil; 68\% yield; ${ }^{1} \mathrm{H}$ NMR: $\delta$ 6.86-6.69 (m, 4H), $4.63(\mathrm{q}, J=6.8 \mathrm{~Hz}, 1 \mathrm{H}), 4.07$ (q, $J=7.2 \mathrm{~Hz}, 2 \mathrm{H}), 3.71$ (s, 3H), 1.51 (d, $J=7.2 \mathrm{~Hz}, 3 \mathrm{H}), 1.19$ (t, $J=7.2 \mathrm{~Hz}, 3 \mathrm{H}) ;{ }^{13} \mathrm{C}$ NMR: $\delta$ 172.1, 160.3, 147.1, 122.7, 120.7.4, 116.7, 112.6, 74.2, 60.9, 55.8, 18.5, 14.0; HRMS (EI) Calcd for $\mathrm{C}_{12} \mathrm{H}_{16} \mathrm{O}_{4}$ : 224.1049; Found 224.1048; 95\% ee [SFC condition: Chiralpak OD-H column, sc $\mathrm{CO}_{2} / \mathrm{i}-\mathrm{PrOH}=94: 6$, flow rate $=2.0 \mathrm{~mL} / \mathrm{min}$, wavelength $=220 \mathrm{~nm}, t_{\mathrm{R}}=4.69 \mathrm{~min}$ for minor isomer, $t_{\mathrm{R}}=5.82 \mathrm{~min}$ for major isomer $][\alpha]_{\mathrm{D}}{ }^{28}=+55.6$ (c 0.9, EtOH) .

\section{(-)-Ethyl 2-(o-phenylphenoxy)propionate (4d)}

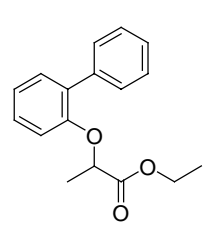

Colorless oil; 83\% yield; ${ }^{1} \mathrm{H}$ NMR: $\delta 7.62$ (d, $J=7.2 \mathrm{~Hz}, 2 \mathrm{H}$ ), 7.42-7.23 (m, 5H), 7.06 (t, $J$ $=7.2 \mathrm{~Hz}, 1 \mathrm{H}), 6.87(\mathrm{~d}, J=8.0 \mathrm{~Hz}, 1 \mathrm{H}), 4.67$ (q, $J=6.8 \mathrm{~Hz}, 1 \mathrm{H}), 4.18$ (q, $J=7.2 \mathrm{~Hz}, 2 \mathrm{H})$, 1.49 (d, $J=6.8 \mathrm{~Hz}, 3 \mathrm{H}), 1.23$ (t, $J=7.2 \mathrm{~Hz}, 3 \mathrm{H}) ;{ }^{13} \mathrm{C}$ NMR: $\delta$ 172.2, 154.6, 138.4, 131.7, 131.2, 129.6, 128.4, 127.9, 126.9, 122.1 114.0, 73.4, 61.1, 18.4, 14.1; HRMS (EI) Calcd for $\mathrm{C}_{17} \mathrm{H}_{18} \mathrm{O}_{3}$ : 270.1256; Found: 270.1250; 99.2\% ee [HPLC condition: Chiralcel OJ + OJ-H column, $n$-Hexane $/ \mathrm{i}-\mathrm{PrOH}=98: 2$, flow rate $=0.6 \mathrm{~mL} / \mathrm{min}$, wavelength $=254 \mathrm{~nm}, t_{\mathrm{R}}=42.90 \mathrm{~min}$ for minor isomer, $t_{\mathrm{R}}=45.85 \mathrm{~min}$ for major isomer $] ;[\alpha]_{\mathrm{D}}{ }^{18}=-21.8\left(c 0.8, \mathrm{CH}_{2} \mathrm{Cl}_{2}\right)$.

\section{(+)-Ethyl 2-(m-tolyloxy)propionate (4e) ${ }^{9}$}

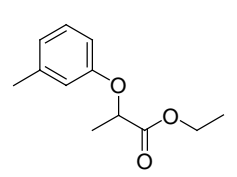

Colorless oil; 79\% yield; ${ }^{1} \mathrm{H}$ NMR: $\delta 7.14(\mathrm{t}, J=8.0 \mathrm{~Hz} 1 \mathrm{H}), 6.78(\mathrm{~d}, J=7.6 \mathrm{~Hz}, 1 \mathrm{H})$, 6.71(s, 1H), 6.66 (d, $J=8.0 \mathrm{~Hz}, 1 \mathrm{H}), 4.73$ (q, $J=6.8 \mathrm{~Hz}, 1 \mathrm{H}), 4.22$ (q, $J=7.2 \mathrm{~Hz}, 2 \mathrm{H}$ ), 2.31(s, 3H), 1.60 (d, $J=6.8 \mathrm{~Hz}, 3 \mathrm{H}), 1.25$ (t, $J=7.2 \mathrm{~Hz}, 3 \mathrm{H}$ ); 99.3\% ee [HPLC condition: Chiralcel OD-H column, $n$-Hexane $/ \mathrm{i}-\mathrm{PrOH}=95: 5$, flow rate $=1.0 \mathrm{~mL} / \mathrm{min}$, wavelength $=$ $210 \mathrm{~nm}, t_{\mathrm{R}}=5.97 \mathrm{~min}$ for minor isomer, $t_{\mathrm{R}}=10.50 \mathrm{~min}$ for major isomer $] ;[\alpha]_{\mathrm{D}}{ }^{18}=+44.1\left(c 1.0, \mathrm{CH}_{2} \mathrm{Cl}_{2}\right)$.

\footnotetext{
7 Kim, K. M.; Kim, H. R.; Chung, K. H.; Song, J. H.; Ryu, E. K.; Syn. Commun. 1994, 24, 1859.

${ }^{8}$ Kim, K. M.; Kim, H. R.; Chung, K. H.; Song, J. H.; Ryu, E. K.; Syn. Commun. 1994, 24, 1859.

9 Azzolina, O.; Ghislandi, V. Farmaco 1993, 48, 1401.
} 


\section{(+)-Ethyl 2-(3-methoxyphenoxy)propionate(4f)}

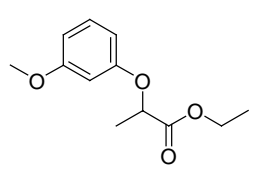

Colorless oil; 71\% yield; ${ }^{1} \mathrm{H}$ NMR: $\delta 7.15$ (t, $\left.J=8.0 \mathrm{~Hz}, 1 \mathrm{H}\right)$; $6.54-6.42(\mathrm{~m}, 3 \mathrm{H}), 4.74$ (q, $J=6.8 \mathrm{~Hz}, 1 \mathrm{H}), 4.21$ (q, $J=7.2 \mathrm{~Hz}, 2 \mathrm{H}), 3.77$ (s, 3H), 1.60 (d, $J=6.8 \mathrm{~Hz}, 3 \mathrm{H}), 1.25$ (t, $J=7.2 \mathrm{~Hz}, 3 \mathrm{H}) ;{ }^{13} \mathrm{C}$ NMR: $\delta$ 172.1, 160.9, 158.9, 129.9, 107.4, 106.9, 101.8, 72.6, 61.2, 55.2, 18.5, 14.1; HRMS (EI) Calcd for $\mathrm{C}_{12} \mathrm{H}_{16} \mathrm{O}_{4}$ : 224.1049; Found: 224.1050; 99\% ee [SFC condition: Chiralpak OD-H column, sc $\mathrm{CO}_{2} / i-\mathrm{PrOH}=90: 10$, flow rate $=2.0 \mathrm{~mL} / \mathrm{min}$, wavelength $=220 \mathrm{~nm}, t_{\mathrm{R}}=3.04 \mathrm{~min}$ for minor isomer, $t_{\mathrm{R}}=3.60 \mathrm{~min}$ for major isomer $] ;[\alpha]_{\mathrm{D}}{ }^{28}=+42.1(c$ $1.0, \mathrm{EtOH})$.

\section{(+)-Ethyl 2-(m-bromophenoxy)propionate (4g)}

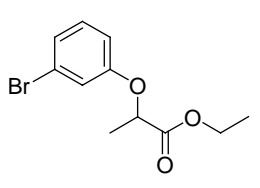

Colorless oil; 78\% yield; ${ }^{1} \mathrm{H}$ NMR: $\delta$ 7.13-7.07 (m, 2H), 7.03 (s, 1H), 6.79-6.77 (m, $1 \mathrm{H}), 4.70$ (q, $J=6.8 \mathrm{~Hz}, 1 \mathrm{H}), 4.21$ (q, $J=7.2 \mathrm{~Hz}, 2 \mathrm{H}), 1.60$ (d, $J=6.8 \mathrm{~Hz}, 3 \mathrm{H}), 1.25$ (t, $J=7.2 \mathrm{~Hz}, 3 \mathrm{H}) ;{ }^{13} \mathrm{C}$ NMR: $\delta 171.9,158.5,130.8,124.9,123.0,118.9,114.1,73.0,61.6$, 18.7, 14.4; HRMS (EI) Calcd for $\mathrm{C}_{11} \mathrm{H}_{13} \mathrm{BrO}_{3}$ : 272.0048; Found: 272.0048; 99\% ee [HPLC condition: Chiralcel OD-H column, $n-\mathrm{Hexane} / \mathrm{i}-\mathrm{PrOH}=98: 2$, flow rate $=1.0 \mathrm{~mL} / \mathrm{min}$, wavelength $=254 \mathrm{~nm}, t_{\mathrm{R}}=6.97 \mathrm{~min}$ for minor isomer, $t_{\mathrm{R}}=8.11 \mathrm{~min}$ for major isomer $] ;[\alpha]_{\mathrm{D}}{ }^{18}=+39.2\left(c 1.0, \mathrm{CH}_{2} \mathrm{Cl}_{2}\right)$.

\section{(+)-Ethyl 2-(m-phenylphenoxy)propionate (4h)}

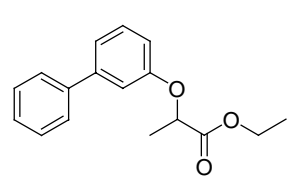

Colorless oil; 82\% yield; ${ }^{1} \mathrm{H}$ NMR: $\delta 7.56$ (d, $J=8.0 \mathrm{~Hz}, 2 \mathrm{H}$ ), 7.44-6.83 (m, 7H), 4.82 (q, $J=6.8 \mathrm{~Hz}, 1 \mathrm{H}), 4.23$ (q, $J=7.2 \mathrm{~Hz}, 2 \mathrm{H}), 1.65$ (d, $J=6.8 \mathrm{~Hz}, 3 \mathrm{H}), 1.25$ (t, $J$ $=7.2 \mathrm{~Hz}, 3 \mathrm{H}) ;{ }^{13} \mathrm{C}$ NMR: $\delta$ 172.2, 158.4, 143.3, 140.9, 128.8, 127.7, 127.2, 119.8, 113.0, 73.0, 61.4, 16.7, 14.3; HRMS (EI) Calcd for $\mathrm{C}_{17} \mathrm{H}_{18} \mathrm{O}_{3}$ : 270.1256; Found: 270.1254; 99\% ee [SFC condition: Chiralcel OD-H column, sc $\mathrm{CO}_{2} / i-\mathrm{PrOH}=90: 10$, flow rate $=2.0$ $\mathrm{mL} / \mathrm{min}$, wavelength $=254 \mathrm{~nm}, t_{\mathrm{R}}=5.01 \mathrm{~min}$ for minor isomer, $t_{\mathrm{R}}=5.80 \mathrm{~min}$ for major isomer $]_{;}[\alpha]_{\mathrm{D}}{ }^{18}=$ $+33.8\left(c\right.$ 1.0, $\left.\mathrm{CH}_{2} \mathrm{Cl}_{2}\right)$.

\section{(+)-Ethyl 2-(p-tolyloxy)propionate (4i) ${ }^{10}$}

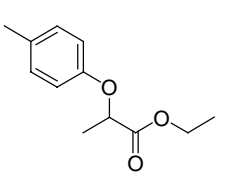

Colorless oil; 88\% yield; ${ }^{1} \mathrm{H}$ NMR: $\delta 7.06$ (d, $\left.J=8.4 \mathrm{~Hz}, 2 \mathrm{H}\right), 6.77$ (d, $J=8.4 \mathrm{~Hz}, 2 \mathrm{H}$ ), 4.70 (q, $J=6.8 \mathrm{~Hz}, 1 \mathrm{H}), 4.20$ (q, $J=6.8 \mathrm{~Hz}, 2 \mathrm{H}), 2.27$ (s, 3H), 1.60 (d, $J=6.8 \mathrm{~Hz}, 3 \mathrm{H}$ ), $1.25(\mathrm{t}, J=6.8 \mathrm{~Hz}, 3 \mathrm{H})$; 99\% ee [HPLC condition: Chiralcel OJ-H column, $n$-Hexane $/ \mathrm{i}-\mathrm{PrOH}=90: 10$, flow rate $=1.0 \mathrm{~mL} / \mathrm{min}$, wavelength $=210 \mathrm{~nm}, t_{\mathrm{R}}=10.25 \mathrm{~min}$ for major isomer, $t_{\mathrm{R}}=19.16 \mathrm{~min}$ for minor isomer]; $[\alpha]_{\mathrm{D}}{ }^{18}=+46.9\left(c 0.75, \mathrm{CH}_{2} \mathrm{Cl}_{2}\right)$.

\section{(+)-Ethyl 2-(4-methoxyphenoxy)propionate(4j) ${ }^{10}$}

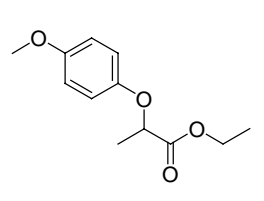

Colorless oil; 78\% yield; ${ }^{1} \mathrm{H}$ NMR: $\delta$ 6.85-6.78 (m, 4H), $4.64(\mathrm{q}, J=6.8 \mathrm{~Hz}, 1 \mathrm{H}), 4.20$ (q, $J=7.2 \mathrm{~Hz}, 2 \mathrm{H}$ ), 3.75 (s, 3H), 1.58 (d, $J=6.8 \mathrm{~Hz}, 3 \mathrm{H}), 1.24$ (t, $J=7.2 \mathrm{~Hz}, 3 \mathrm{H}$ ); $99 \%$ ee [SFC condition: Chiralpak OJ-H column, sc $\mathrm{CO}_{2} / i-\mathrm{PrOH}=94: 6$, flow rate $=2.0$ $\mathrm{mL} / \mathrm{min}$, wavelength $=220 \mathrm{~nm}, t_{\mathrm{R}}=4.39 \mathrm{~min}$ for minor isomer, $t_{\mathrm{R}}=4.69$ min for major isomer]; $[\alpha]_{\mathrm{D}}^{28}=+57.0($ c 1.6, EtOH).

\section{(+)-Ethyl 2-(p-tert-butylphenoxy)propionate (4k)}

Colorless oil; 70\% yield; ${ }^{1} \mathrm{H}$ NMR: $\delta 7.19$ (d, $J=9.0 \mathrm{~Hz}, 2 \mathrm{H}$ ), 6.73 (d, $J=9.0 \mathrm{~Hz}, 2 \mathrm{H}$ ), 4.63 (q, $J=6.9 \mathrm{~Hz}$,

\footnotetext{
${ }^{10}$ Kato, D. I.; Mitsuda, S.; Ohta, H. J. Org. Chem. 2003, 68, 7234.
} 


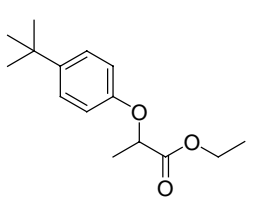

1H), 4.14 (q, $J=7.2 \mathrm{~Hz}, 2 \mathrm{H}), 1.52$ (d, $J=6.9 \mathrm{~Hz}, 3 \mathrm{H}), 1.20-1.14$ (m, 12H); ${ }^{13} \mathrm{C}$ NMR: $\delta$ 172.4, 155.4, 144.2, 126.3, 114.6, 72.8, 61.2, 34.1, 31.5, 18.6,14.2; HRMS (EI) Calcd for $\mathrm{C}_{15} \mathrm{H}_{22} \mathrm{O}_{3}$ : 250.1569, Found: 250.1568; 98\% ee [SFC condition: Chiralpak AD-H column, sc $\mathrm{CO}_{2} / \mathrm{i}-\mathrm{PrOH}=85: 15$, flow rate $=2.0 \mathrm{~mL} / \mathrm{min}$, wavelength $=220 \mathrm{~nm}, t_{\mathrm{R}}=$ $2.01 \mathrm{~min}$ for minor isomer, $t_{\mathrm{R}}=2.18 \mathrm{~min}$ for major isomer $]$; $[\alpha]_{\mathrm{D}}^{18}=+42.0\left(\mathrm{c} 1.0, \mathrm{CH}_{2} \mathrm{Cl}_{2}\right)$.

\section{(R)-(+)-Ethyl 2-(p-chlorophenoxy)propionate (4l) ${ }^{11}$}

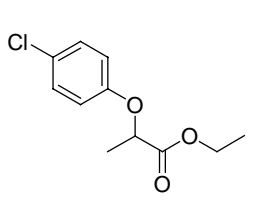

Colorless oil; 83\% yield; ${ }^{1} \mathrm{H}$ NMR: $\delta 7.22$ (d, $\left.J=8.8 \mathrm{~Hz}, 2 \mathrm{H}\right), 6.97$ (d, $\left.J=8.8 \mathrm{~Hz}, 2 \mathrm{H}\right)$, 4.69 (q, $J=6.8 \mathrm{~Hz}, 1 \mathrm{H}), 4.21$ (q, $J=7.2 \mathrm{~Hz}, 2 \mathrm{H}), 1.60$ (d, $J=6.8 \mathrm{~Hz}, 3 \mathrm{H}), 1.24$ (t, $J=$ $7.2 \mathrm{~Hz}, 3 \mathrm{H}) ;{ }^{13} \mathrm{C}$ NMR: $\delta$ 170.8, 155.3, 128.4, 125.4, 115.5, 72.0, 60.3, 17.4, 13.1;

HRMS Anal. Calcd for $\mathrm{C}_{11} \mathrm{H}_{13} \mathrm{ClO}_{3}$ : 228.0553; Found: 228.0552; 99.1\% ee [HPLC condition: Chiralcel OJ-H column, $n$-Hexane $/ \mathrm{i}-\mathrm{PrOH}=90: 10$, flow rate $=1.0 \mathrm{~mL} / \mathrm{min}$, wavelength $=210$ $\mathrm{nm}, t_{\mathrm{R}}=8.27 \mathrm{~min}$ for major isomer, $t_{\mathrm{R}}=11.97 \mathrm{~min}$ for minor isomer $]$; $[\alpha]_{\mathrm{D}}{ }^{18}=+47.6\left(c 1.0, \mathrm{CH}_{2} \mathrm{Cl}_{2}\right)$.

\section{(+)-Ethyl 2-(4-bromophenoxy)propionate (4m) ${ }^{\mathbf{1 0}}$}

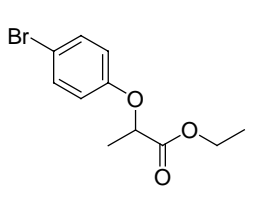

Colorless oil; 85\% yield; ${ }^{1} \mathrm{H}$ NMR: $\delta 7.36$ (d, $\left.J=8.8 \mathrm{~Hz}, 2 \mathrm{H}\right), 6.75$ (d, $\left.J=8.8 \mathrm{~Hz}, 2 \mathrm{H}\right)$, 4.69 (q, $J=6.8 \mathrm{~Hz}, 1 \mathrm{H}), 4.20$ (q, $J=7.2 \mathrm{~Hz}, 2 \mathrm{H}), 1.61$ (d, $J=6.8 \mathrm{~Hz}, 3 \mathrm{H}), 1.24(\mathrm{t}, J=$ $7.2 \mathrm{~Hz}, 3 \mathrm{H}$ ); 99.6\% ee [SFC condition: Chiralcel OD-H column, sc $\mathrm{CO}_{2} / i-\mathrm{PrOH}=95: 5$, flow rate $=2.0 \mathrm{~mL} / \mathrm{min}$, wavelength $=220 \mathrm{~nm}, t_{\mathrm{R}}=3.55 \mathrm{~min}$ for major isomer, $t_{\mathrm{R}}=3.96$ min for minor isomer $]$; $[\alpha]_{\mathrm{D}}^{18}=+47.1$ (c 1.0, EtOH).

\section{(+)-Methyl 4-(1-ethoxy-1-oxopropan-2-yloxy)benzoate (4n)}

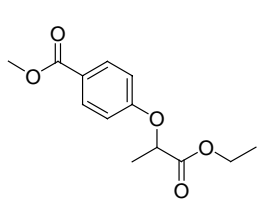

Colorless oil; 62\% yield; ${ }^{1} \mathrm{H}$ NMR: $\delta 7.97$ (d, $\left.J=9.0 \mathrm{~Hz}, 2 \mathrm{H}\right), 6.89$ (d, $\left.J=9.0 \mathrm{~Hz}, 2 \mathrm{H}\right)$, 4.81 (q, $J=6.9 \mathrm{~Hz}, 1 \mathrm{H}), 4.22$ (q, $J=7.2 \mathrm{~Hz}, 2 \mathrm{H}), 3.88$ (s, 3H), 1.64 (d, $J=6.8 \mathrm{~Hz}, 3 \mathrm{H})$, $1.24(\mathrm{t}, J=7.2 \mathrm{~Hz}, 3 \mathrm{H}) ;{ }^{13} \mathrm{C}$ NMR: $\delta 171.7,166.8,161.5,131.8,123.6,114.8,72.8$, 61.6, 52.0, 18.6, 14.3; MS (EI) m/z $252\left(\mathrm{M}^{+}, 50\right)$; Anal. Calcd for $\mathrm{C}_{13} \mathrm{H}_{16} \mathrm{O}_{5}$ : C 61.90, H 6.39; Found: C 61.87, H 6.73; 99\% ee [SFC condition: Chiralpak OJ-H column, sc $\mathrm{CO}_{2} / i-\mathrm{PrOH}=95: 5$, flow rate $=2.0 \mathrm{~mL} / \mathrm{min}$, wavelength $=254 \mathrm{~nm}, t_{\mathrm{R}}=4.15 \mathrm{~min}$ for minor isomer, $t_{\mathrm{R}}=4.82$ min for major isomer $][\alpha]_{\mathrm{D}}{ }^{26}=+47.2(c 0.3, \mathrm{EtOH})$.

\section{(+)-Ethyl 2-(2,4-dimethylphenoxy)propionate (40)}

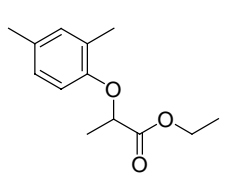

Colorless oil; 80\% yield; ${ }^{1} \mathrm{H}$ NMR: $\delta 6.97$ (s, 1H), 6.89 (d, $\left.J=8.0 \mathrm{~Hz}, 1 \mathrm{H}\right), 6.60$ (d, $J=$ $8.0 \mathrm{~Hz}, 1 \mathrm{H}), 4.69$ (q, $J=6.8 \mathrm{~Hz}, 1 \mathrm{H}), 4.21$ (q, $J=7.2 \mathrm{~Hz}, 2 \mathrm{H}), 2.26$ (s, 3H), 2.25 (s, 3H), $1.62(\mathrm{~d}, J=6.8 \mathrm{~Hz}, 3 \mathrm{H}), 1.26(\mathrm{t}, J=7.2 \mathrm{~Hz}, 3 \mathrm{H}) ;{ }^{13} \mathrm{C}$ NMR: $\delta 172.7,154.1,132.0,130.7$, 127.6, 127.0, 112.5, 73.5, 61.3, 20.7, 18.9, 16.4, 14.3; MS (EI) m/z $222\left(\mathrm{M}^{+}, 60\right)$; Anal. Calcd for $\mathrm{C}_{13} \mathrm{H}_{18} \mathrm{O}_{3}$ : C 70.24, $\mathrm{H}$ 8.16; Found: C 69.87, H 7.73; 99\% ee [HPLC condition: Chiralcel OB column, $n$-hexane $/ \mathrm{i}$-PrOH $=99: 1$, flow rate $=0.8 \mathrm{~mL} / \mathrm{min}$, wavelength $=230 \mathrm{~nm}, t_{\mathrm{R}}=12.80$ min for minor isomer, $t_{\mathrm{R}}=15.34 \mathrm{~min}$ for major isomer $\left.] ; \alpha\right]_{\mathrm{D}}{ }^{26}=+30.0(c \mathrm{c} .7, \mathrm{EtOH})$.

\section{(+)-Ethyl 2-(3,5-diphenylphenoxy)propionate (4p)}

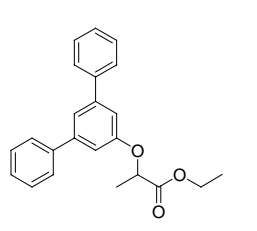

Colorless oil; 84\% yield; ${ }^{1} \mathrm{H}$ NMR: $\delta 7.61$ (d, $\left.J=7.6 \mathrm{~Hz}, 4 \mathrm{H}\right), 7.46-7.36$ (m, 7H), 7.09 (s, 2H), 4.88 (q, $J=6.8 \mathrm{~Hz}, 1 \mathrm{H}), 4.29-4.21$ (m, 2H), 1.67 (d, $J=6.8 \mathrm{~Hz}, 3 \mathrm{H}), 1.26$ (t, $J=7.2$ $\mathrm{Hz}, 3 \mathrm{H}) ;{ }^{13} \mathrm{C}$ NMR: $\delta 171.1,157.0,141.8,139.8,128.8,127.7,126.4,126.1,119.5,113.3$,

${ }^{11}$ Bettoni, G.; Loiodice, F.; Tortorella, V.; Conte-Camerino, D.; Mambrini, M.; Ferrannini, E.; Bryant, S.-H. J. Med. Chem. 1987, 30, 1267. 
112.7, 71.7, 60.2, 17.5, 13.1; HRMS (EI) Calcd for $\mathrm{C}_{23} \mathrm{H}_{22} \mathrm{O}_{3}$ : 346.1569; Found: 346.1559; 99\% ee [HPLC condition: Chiralpak AD-H column, $n$-Hexane $/ i-\mathrm{PrOH}=98: 2$, flow rate $=1.0 \mathrm{~mL} / \mathrm{min}$, wavelength $=254$ $\mathrm{nm}, t_{\mathrm{R}}=7.33 \mathrm{~min}$ for minor isomer, $t_{\mathrm{R}}=8.69 \mathrm{~min}$ for major isomer $]$; $[\alpha]_{\mathrm{D}}{ }^{18}=+36.3\left(c 0.4, \mathrm{CH}_{2} \mathrm{Cl}_{2}\right)$.

\section{(R)-(-)-Ethyl 2-(naphthalen-1-yloxy)propionate (4q) ${ }^{12}$}

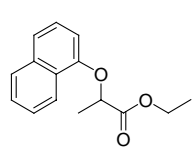

Colorless oil; 77\% yield; ${ }^{1} \mathrm{H}$ NMR: $\delta 8.36(\mathrm{t}, J=6.8 \mathrm{~Hz}, 1 \mathrm{H}), 7.80(\mathrm{t}, J=4.8 \mathrm{~Hz}, 1 \mathrm{H}$ ), 7.50-7.44 (m, 3H), 7.32 (t, $J=8.0 \mathrm{~Hz}, 1 \mathrm{H}), 6.70$ (t, $J=8.0 \mathrm{~Hz}, 1 \mathrm{H}), 4.94$ (q, $J=6.8 \mathrm{~Hz}$,

1H), 4.23 (q, $J=7.2 \mathrm{~Hz}, 2 \mathrm{H}), 1.76$ (d, $J=6.8 \mathrm{~Hz}, 3 \mathrm{H}), 1.24(\mathrm{t}, J=7.2 \mathrm{~Hz}, 3 \mathrm{H}) ; 99.1 \%$ ee

[HPLC condition: Chiralcel OD-H column, $n$-Hexane $/ \mathrm{i}-\mathrm{PrOH}=93: 7$, flow rate $=1.0 \mathrm{~mL} / \mathrm{min}$, wavelength $=210 \mathrm{~nm}, t_{\mathrm{R}}=5.78 \mathrm{~min}$ for $(S)$-enantiomer, $t_{\mathrm{R}}=8.52 \mathrm{~min}$ for $(R)$-enantiomer $]$; $[\alpha]_{\mathrm{D}}{ }^{18}=-35.0$ (c 0.4, $\left.\mathrm{CHCl}_{3}\right)\left[\text { lit: }[\alpha]_{\mathrm{D}}{ }^{20}=-38.7\left(c 4.0, \mathrm{CHCl}_{3}\right) \text { for }(R)\right]^{11}$

\section{(R)-(+)-Ethyl 2-(naphthalen-2-yloxy)propionate (4r) ${ }^{12}$}

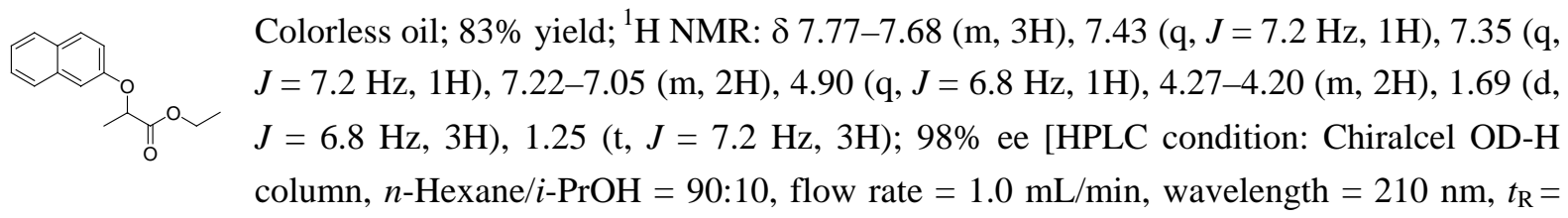
6.69 min for $(R)$-enantiomer, $t_{\mathrm{R}}=7.44 \mathrm{~min}$ for $(S)$-enantiomer $]$; $[\alpha]_{\mathrm{D}}{ }^{18}=+101\left(c 1.4\right.$, EtOH) $\left[\right.$ lit: $[\alpha]_{\mathrm{D}}{ }^{20}=$ -105 (c 2.5, EtOH) for $(S)]^{12}$

\section{(+)-Ethyl 2-(3-phenylpropoxy)propionate (4s)}

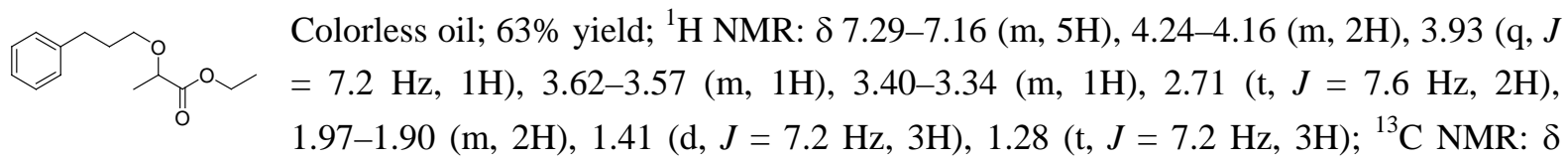

173.7, 142.1, 128.7, 126.0, 75.3, 73.0, 69.6, 61.0, 32.4, 31.5, 18.9, 14.5; HRMS (ESI) Calcd for [ $\mathrm{C}_{13} \mathrm{H}_{18} \mathrm{O}_{3}+\mathrm{Na}$ ]: 259.1310, Found: 259.1305; 59\% ee [HPLC condition: Chiralcel OJ-H column, $n$-Hexane $/ \mathrm{i}-\mathrm{PrOH}=99: 1$, flow rate $=1.0 \mathrm{~mL} / \mathrm{min}$, wavelength $=210 \mathrm{~nm}, t_{\mathrm{R}}=10.02 \mathrm{~min}$ for minor isomer, $t_{\mathrm{R}}=11.00$ min for major isomer $] ;[\alpha]_{\mathrm{D}}{ }^{18}=+18.8(c$ 1.0, EtOH).

\section{$(R)-(+)-E t h y l ~ 2-\left(\right.$ cinnamyloxy)propionate $(4 t){ }^{13}$}

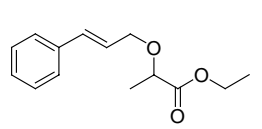

Colorless oil; 85\% yield; ${ }^{1} \mathrm{H}$ NMR: $\delta$ 7.39-7.22 (m, 5H), $6.61(\mathrm{~d}, J=16.0 \mathrm{~Hz}, 1 \mathrm{H})$, 6.33-6.26 (m, 1H), 4.32-4.04 (m, 5H), 1.44 (d, $J=6.8 \mathrm{~Hz}, 3 \mathrm{H}), 1.38$ (t, $J=7.2 \mathrm{~Hz}, 3 \mathrm{H}$ ); 61\% ee [HPLC condition: Chiralpak AD-H column, $n$-Hexane/i-PrOH = 99:1, flow rate $=1.0 \mathrm{~mL} / \mathrm{min}$, wavelength $=254 \mathrm{~nm}, t_{\mathrm{R}}=7.07 \mathrm{~min}$ for minor isomer, $t_{\mathrm{R}}=7.83 \mathrm{~min}$ for major isomer $]$; $[\alpha]_{\mathrm{D}}^{18}=+35.5$ (c 1.0, EtOH).

\section{(R)-(-)-Methyl 2-phenoxy-2-phenylacetate (4u) ${ }^{14}$}

Colorless oil; 71\% yield; ${ }^{1} \mathrm{H}$ NMR: $\delta 7.27$ (t, $\left.J=8.0 \mathrm{~Hz}, 2 \mathrm{H}\right), 7.42-7.25$ (m, 5H), 7.05-6.95<smiles>COC(=O)C(OC)c1ccccc1</smiles>
(m, 3H), 5.65 (s, 1H), 3.74 (s, 3H); 10\% ee [HPLC condition: Chiralcel OD-H column, $n$-Hexane $/ \mathrm{i}-\mathrm{PrOH}=97: 3$, flow rate $=1.0 \mathrm{~mL} / \mathrm{min}$, wavelength $=210 \mathrm{~nm}, t_{\mathrm{R}}=7.1 \mathrm{~min}$ for $(R)$-enantiomer, $t_{\mathrm{R}}=16.9$ min for $(S)$-enantiomer]; $[\alpha]_{\mathrm{D}}{ }^{18}=-8.4\left(c 0.5, \mathrm{CHCl}_{3}\right)\left[\right.$ lit: $[\alpha]_{\mathrm{D}}{ }^{22}=+48.5(c 1.51$, $\mathrm{CHCl}_{3}$ ) for $\left.(S)\right] .^{14}$

\footnotetext{
12 Burkard, U.; Effenberger, F. Chem. Ber. 1986, 119, 1594.

13 Aurich, H. G.; Biesemeier, F. Synthesis 1995, 9, 1171.

14 Shintou, T.; Mukaiyama, T. J. Am. Chem. Soc. 2004, 126, 7359.
} 


\section{(R)-(+)-Methyl 2-phenoxypropionate (4v) ${ }^{15}$}

Colorless oil; 70\% yield; ${ }^{1} \mathrm{H}$ NMR: $\delta 7.28$ (t, $\left.J=8.0 \mathrm{~Hz}, 2 \mathrm{H}\right), 6.97$ (t, $\left.J=7.6 \mathrm{~Hz}, 1 \mathrm{H}\right), 7.27$<smiles>CCOC(=O)C(C)Oc1ccccc1</smiles>
(d, $J=8.0 \mathrm{~Hz}, 2 \mathrm{H}$ ), 4.78 (q, $J=6.8 \mathrm{~Hz}, 1 \mathrm{H}$ ), 3.76 (s, 3H), 1.63 (d, $J=6.8 \mathrm{~Hz}, 3 \mathrm{H}$ ); $98 \%$ ee [HPLC condition: Chiralcel OJ-H column, $n$-Hexane $/ \mathrm{i}-\mathrm{PrOH}=90: 10$, flow rate $=1.0$ $\mathrm{mL} / \mathrm{min}$, wavelength $=210 \mathrm{~nm}, t_{\mathrm{R}}=12.64 \mathrm{~min}$ for $(R)$-enantiomer, $t_{\mathrm{R}}=19.82 \mathrm{~min}$ for (S)-enantiomer]; $[\alpha]_{\mathrm{D}}{ }^{18}=+41.5\left(c 0.4, \mathrm{CHCl}_{3}\right)$ [lit: $[\alpha]_{\mathrm{D}}{ }^{26}=+41.2\left(c 0.58, \mathrm{CHCl}_{3}\right)$ for $(R)$ with $97 \%$ ee]. ${ }^{15}$

\section{(+)-tert-Butyl 2-phenoxypropionate (4w)}

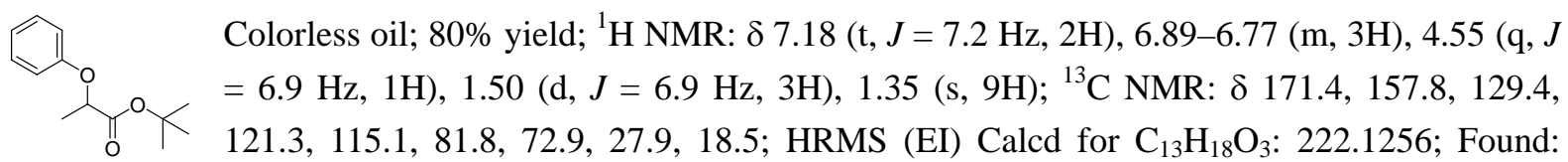
222.1255; 99\% ee [HPLC condition: Chiralcel OD-H column, $n$-Hexane/i-PrOH $=99.3: 0.7$, flow rate $=1.0$ $\mathrm{mL} / \mathrm{min}$, wavelength $=210 \mathrm{~nm}, t_{\mathrm{R}}=8.32 \mathrm{~min}$ for minor isomer, $t_{\mathrm{R}}=9.66 \mathrm{~min}$ for major isomer $]_{;}[\alpha]_{\mathrm{D}}{ }^{18}=$ $+54.3\left(\right.$ c 1.0, $\left.\mathrm{CH}_{2} \mathrm{Cl}_{2}\right)$.

\section{General Procedure for Hydrolysis of 2-Aryloxypropionates}

A solution of $4 \mathbf{a}(260 \mathrm{mg}, 1.3 \mathrm{mmol})$ in EtOH $(3 \mathrm{~mL})$ was added to an aqueous solution of potassium hydroxide ( $5 \mathrm{~mL}, 1.25 \mathrm{M}$ ) at $0{ }^{\circ} \mathrm{C}$. After being stirred for $7 \mathrm{~h}$, the reaction mixture was acidified by $3 \mathrm{M}$ hydrochloric acid and extracted with EtOAc. The organic layer was washed with brine, dried over anhydrous sodium sulfate, and concentrated in vacuum to give 2-phenoxy propanoic acid (5a) (226 mg, quant.) as a white solid. The analytical data for phenyloxypropionic acid are listed below.

\section{Analytical Data for 2-Aryloxypropionic Acids}

\section{(R)-(+)-2-Phenoxypropionic acid (5a) ${ }^{16}$}

$$
\begin{aligned}
& \text { White solid, mp } 85-87^{\circ} \mathrm{C} \text {; } 100 \% \text { yield; }{ }^{1} \mathrm{H} \text { NMR: } \delta 7.30(\mathrm{t}, J=7.6 \mathrm{~Hz}, 2 \mathrm{H}), 7.01(\mathrm{t}, J=7.6 \\
& \mathrm{Hz}, 1 \mathrm{H}), 6.90(\mathrm{t}, J=7.6 \mathrm{~Hz}, 2 \mathrm{H}), 4.80(\mathrm{q}, J=6.8 \mathrm{~Hz}, 1 \mathrm{H}) ; 1.67(\mathrm{~d}, J=6.8 \mathrm{~Hz}, 3 \mathrm{H}) ;[\alpha]_{\mathrm{D}}{ }^{19}= \\
& +38.9(c 1.3, \mathrm{EtOH})\left[\mathrm{lit}[\alpha]_{\mathrm{D}}{ }^{25}=+39.8(c 1, \mathrm{EtOH}) \text { for }(R)\right]{ }^{16}
\end{aligned}
$$

\section{(R)-(+)-2-(4-chlorophenoxy)propionic acid (5l) ${ }^{11}$}

White solid; mp $102-104{ }^{\circ} \mathrm{C} ; 100 \%$ yield; ${ }^{1} \mathrm{H}$ NMR: $\delta 7.24(\mathrm{~d}, J=8.8 \mathrm{~Hz}, 2 \mathrm{H}), 6.82(\mathrm{~d}, J=$
$8.8 \mathrm{~Hz}, 2 \mathrm{H}), 4.75(\mathrm{q}, J=6.4 \mathrm{~Hz}, 1 \mathrm{H}), 1.66(\mathrm{~d}, J=6.8 \mathrm{~Hz}, 3 \mathrm{H}) ;[\alpha]_{\mathrm{D}}{ }^{19}=+35.3$ (с 1.6,

\footnotetext{
15 Kato, D. I.; Mitsuda, S.; Ohta, H. Org. Lett. 2002, 4, 371.

${ }^{16}$ (a) Perron Y. G.; Minor, W. F.; Holdrege, C. T.; Gottstein, W. J.; Godfrey, J. C.; Crast, L. B.; Babel, R. B.; Cheney, L. C. J. Am. Chem. Soc. 1960, 82, 3934. (b) Tottie, L.; Baeckström, P.; Moberg, C. J. Org. Chem. 1992, 57, 6579.
} 
5. NMR Spectra for New O-H Insertion Products

(+)-Ethyl 2-(2-methoxyphenoxy)propionate(4c)

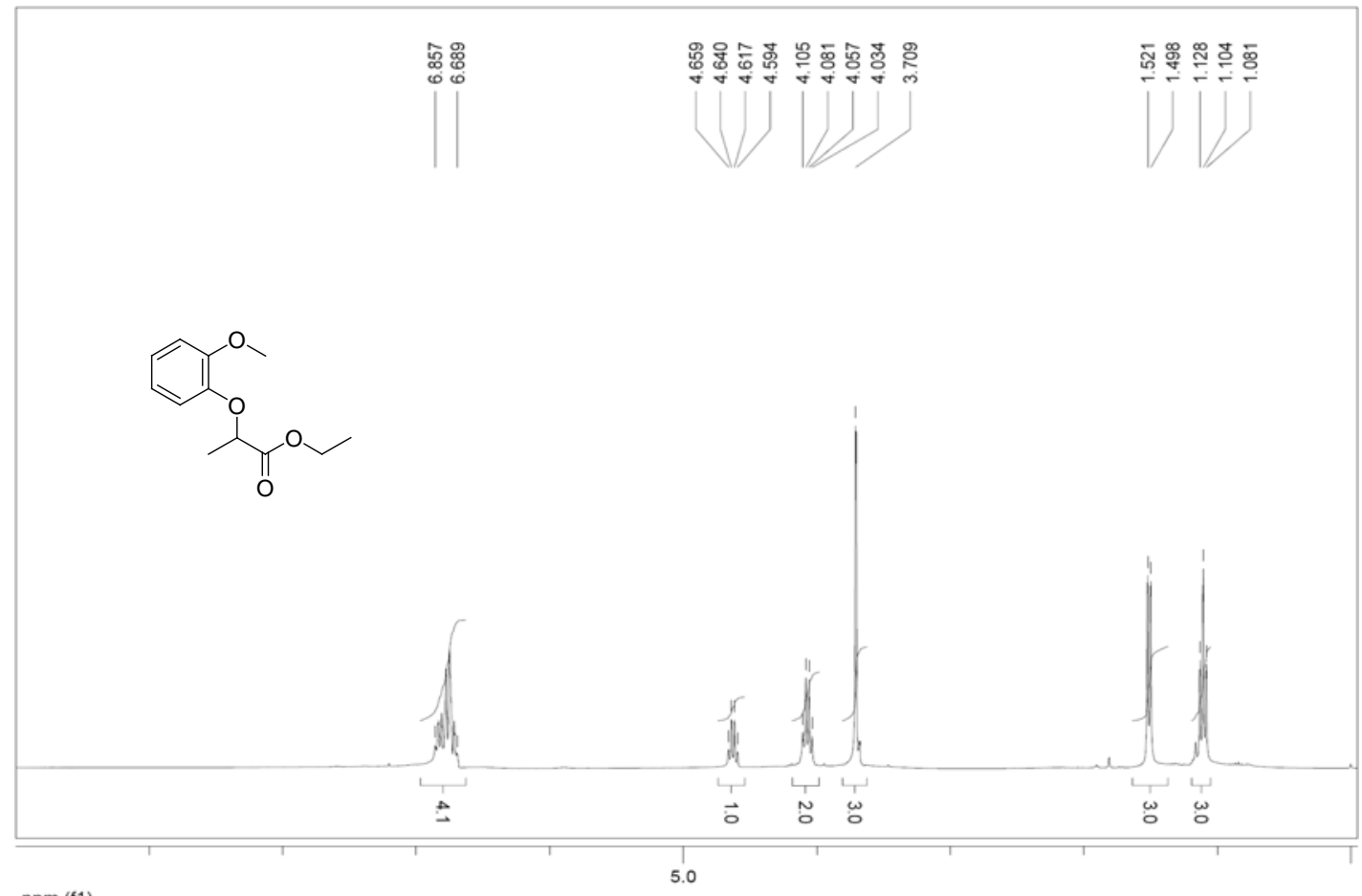

ppm (f1)

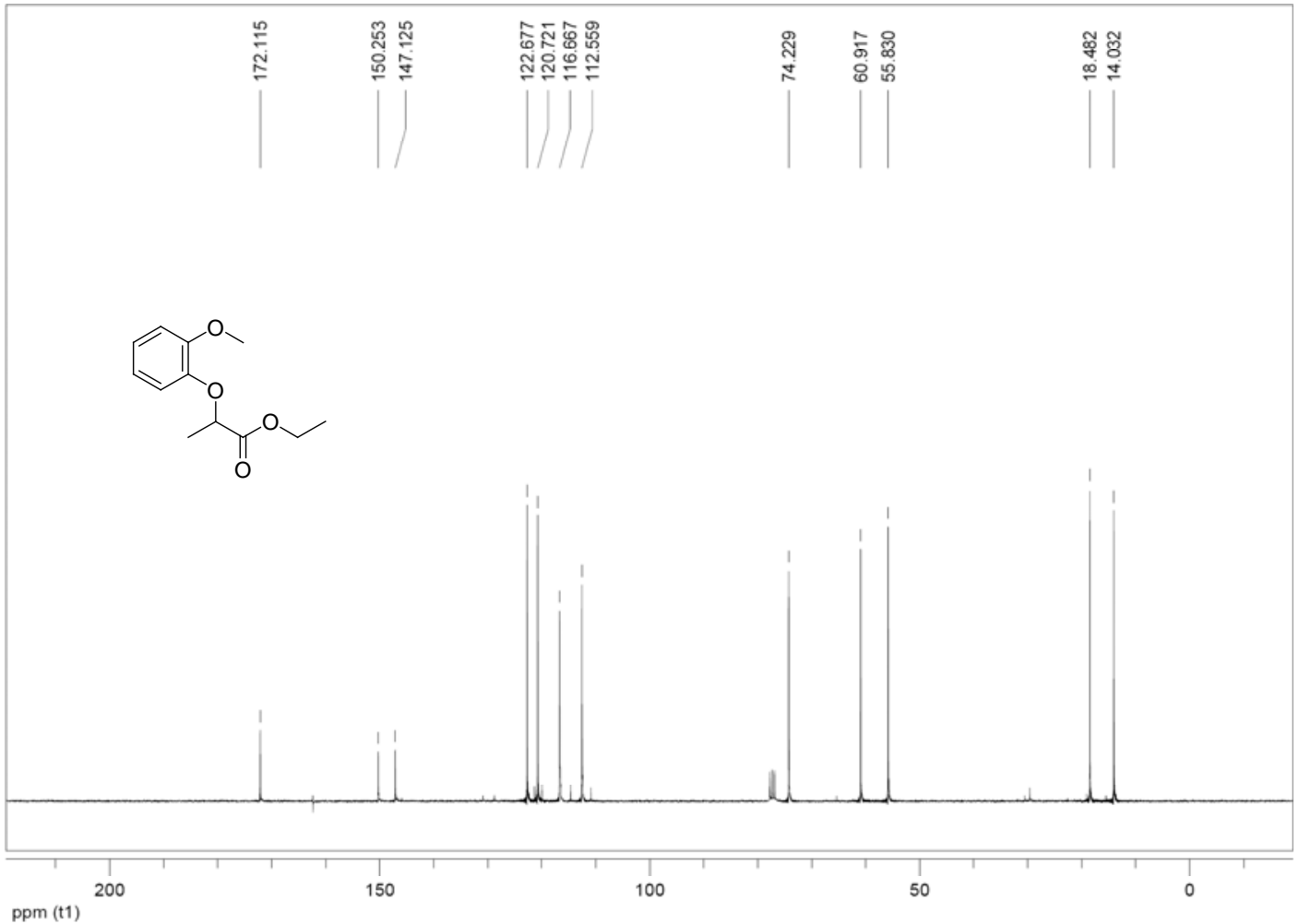


(-)-Ethyl 2-(2-phenylphenoxy)propionate (4d)
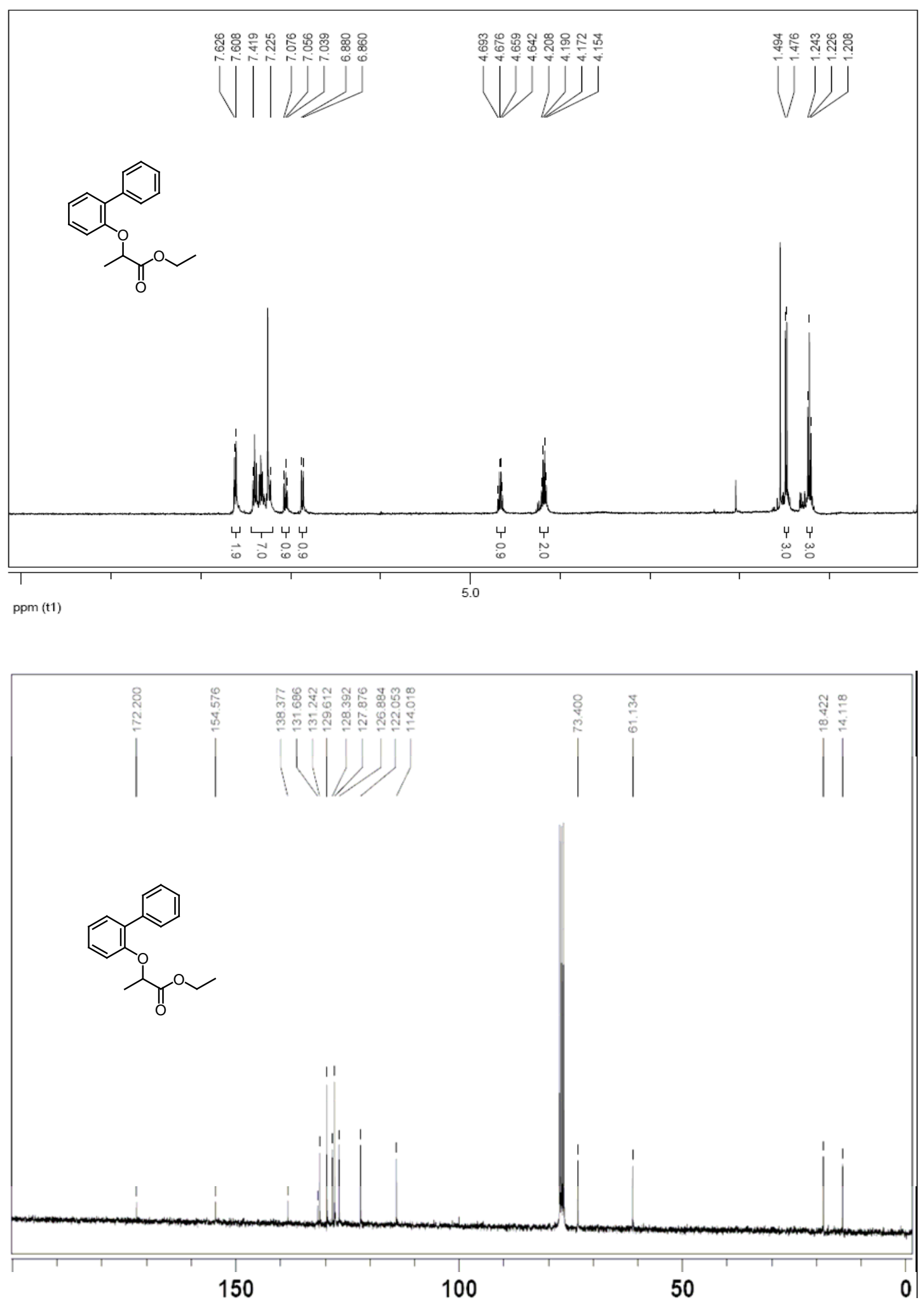

S8 
(+)-Ethyl 2-(3-methoxyphenoxy)propionate(4f)
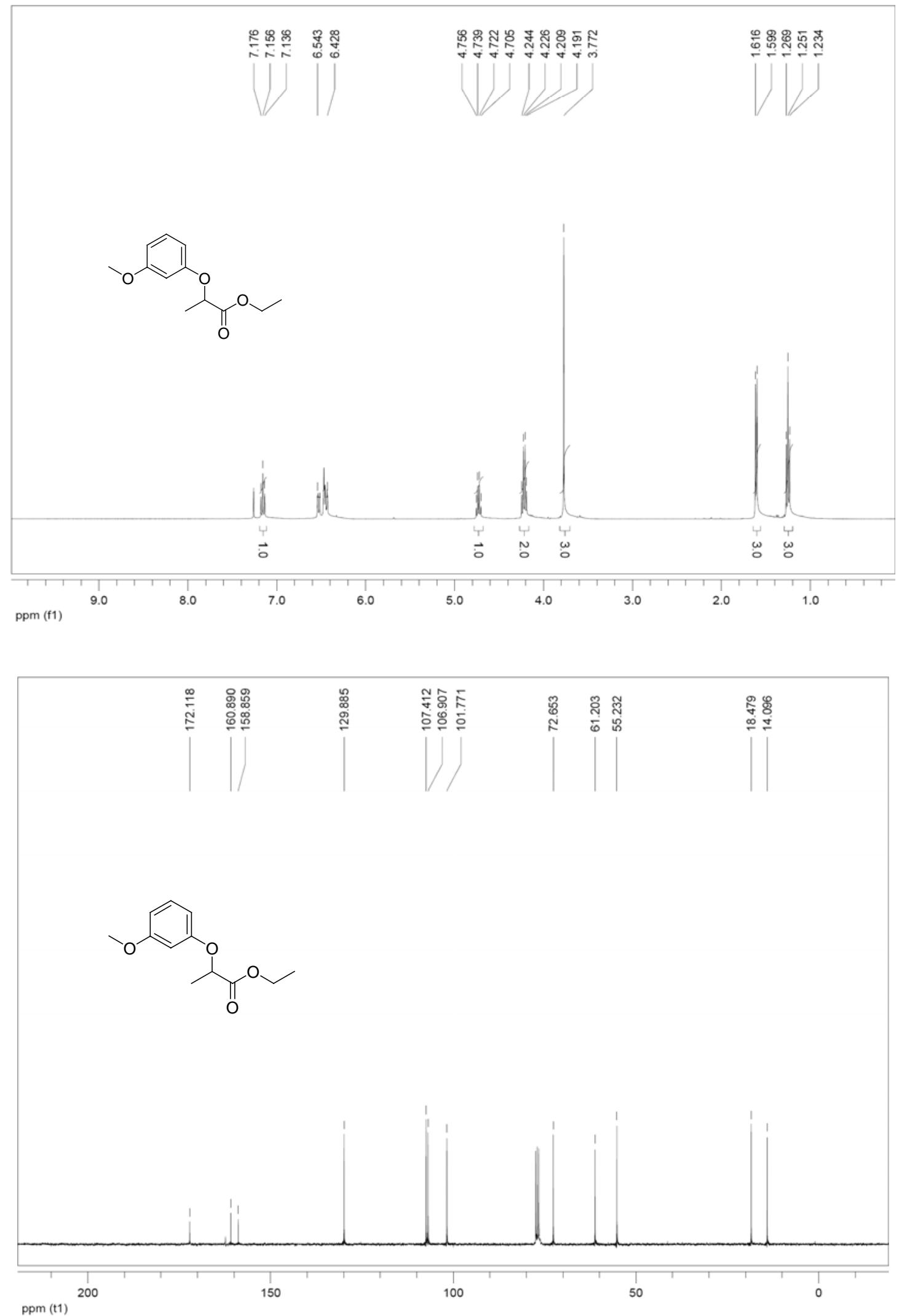
(+)-Ethyl 2-(3-bromophenoxy)propionate(4g)

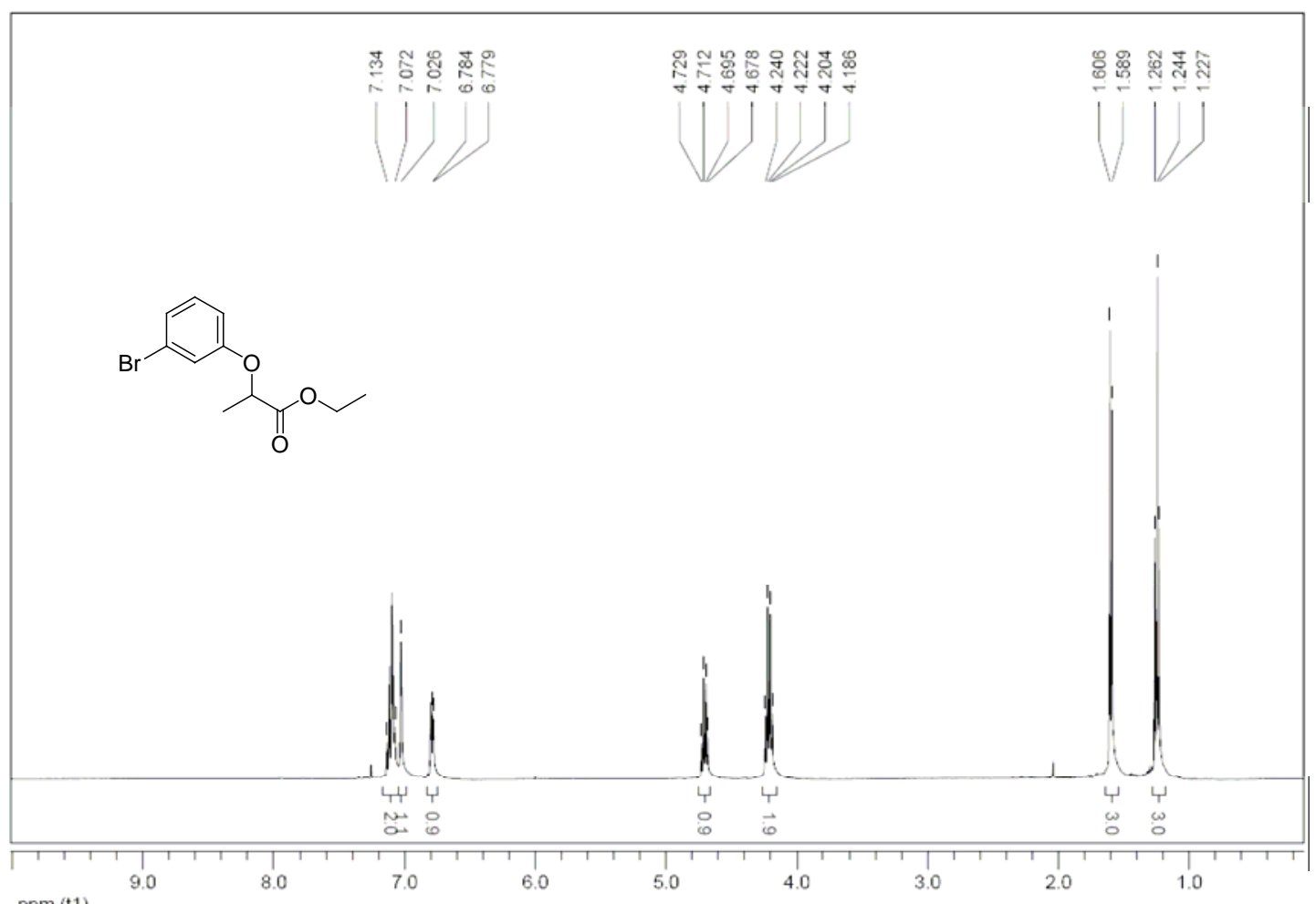

ppm (t1)
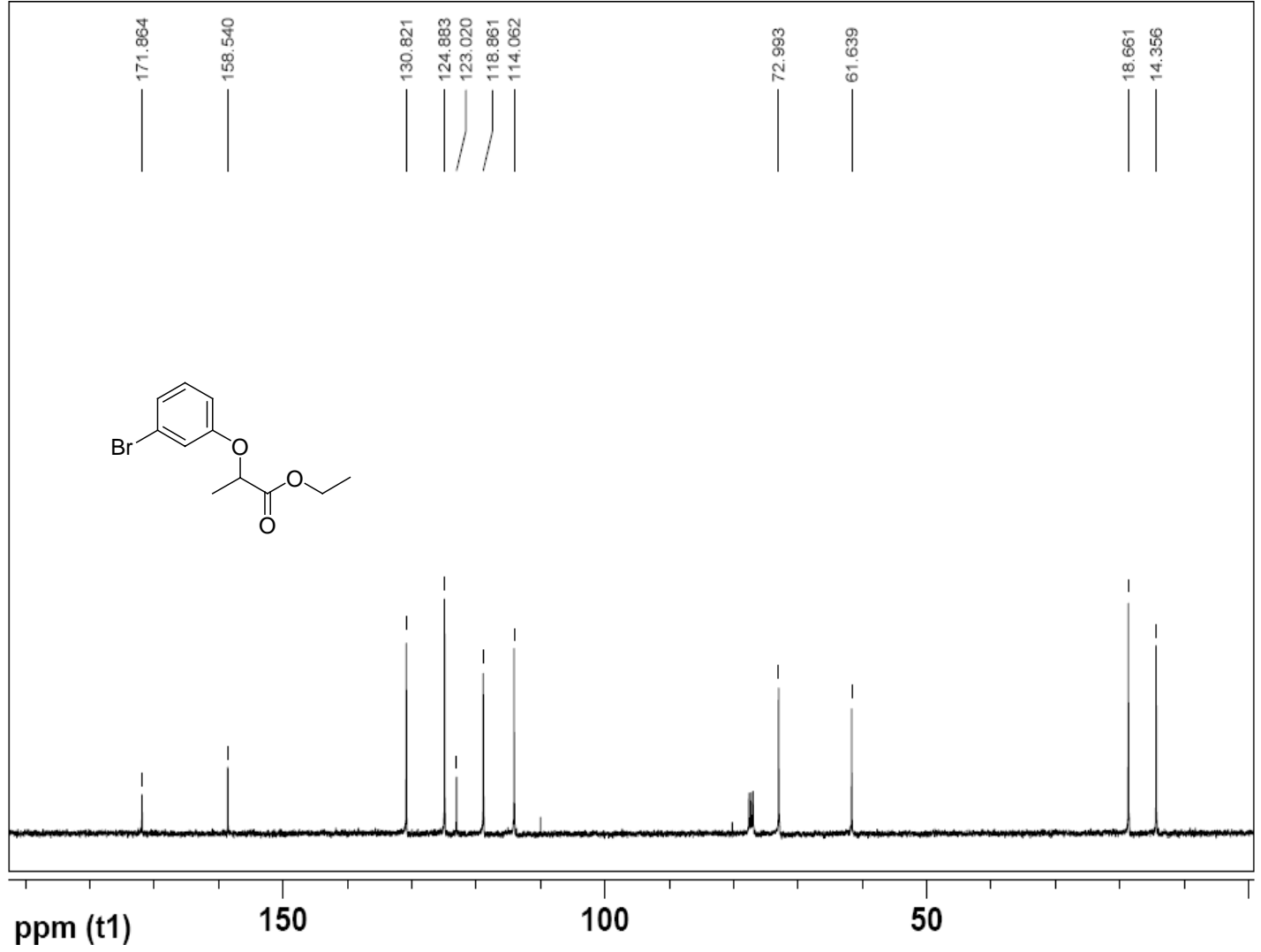
(+)-Ethyl 2-(3-phenylphenoxy)propionate (4h)
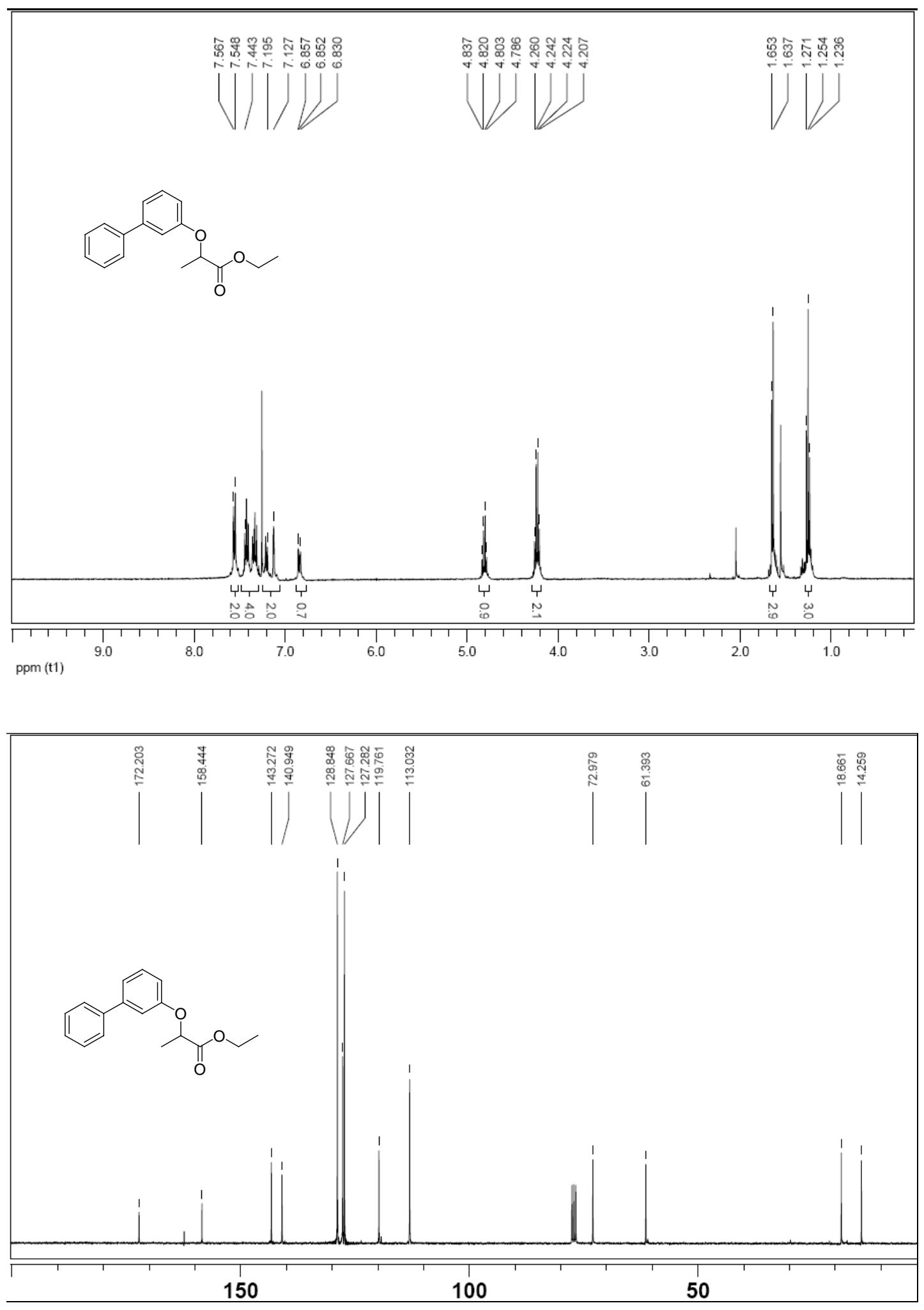
(+)-Ethyl 2-(4-tert-butylphenoxy)propionate(4k)
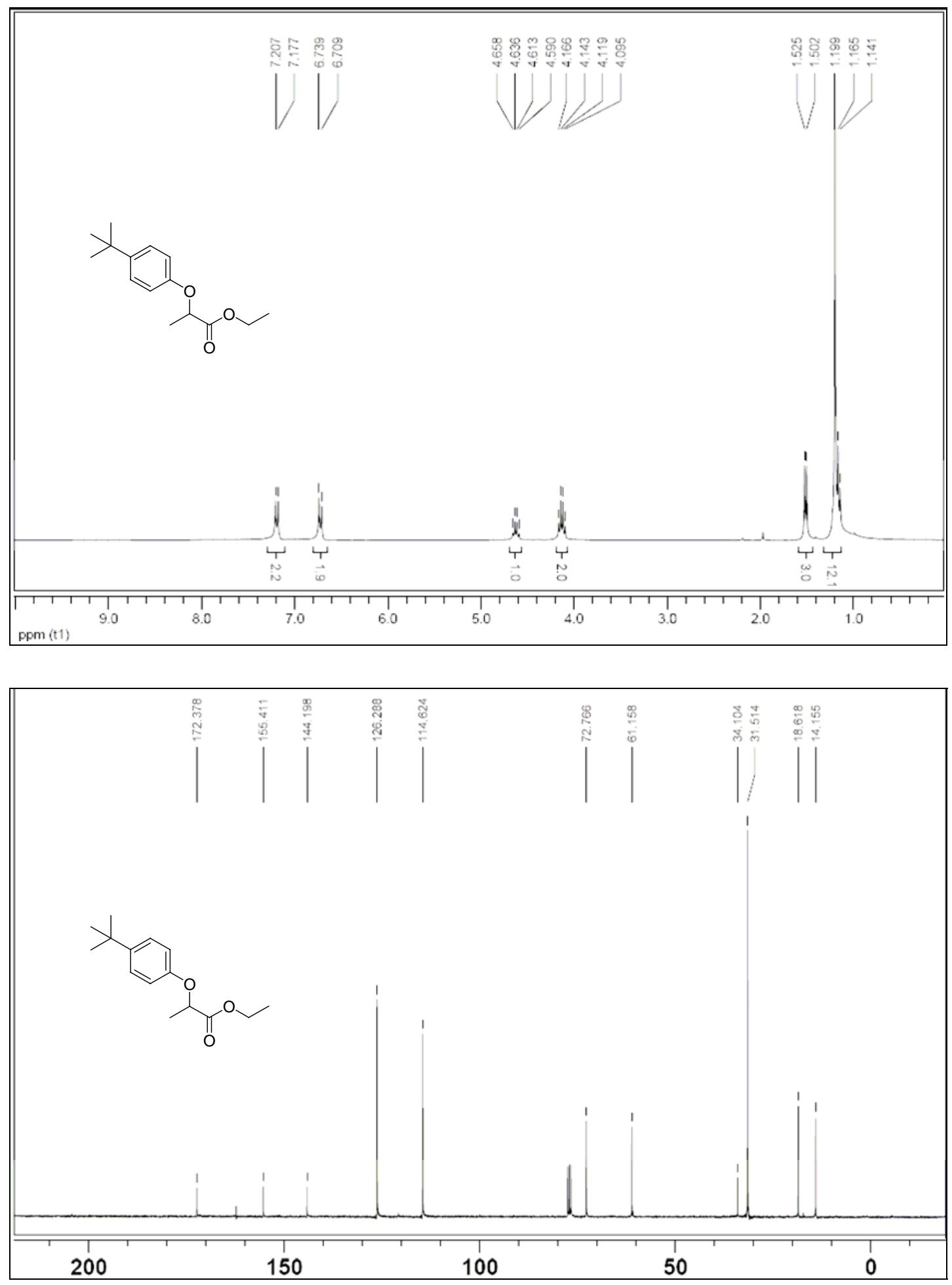

S12 
(+)-Ethyl 2-(p-chlorophenoxy)propionate (4l)
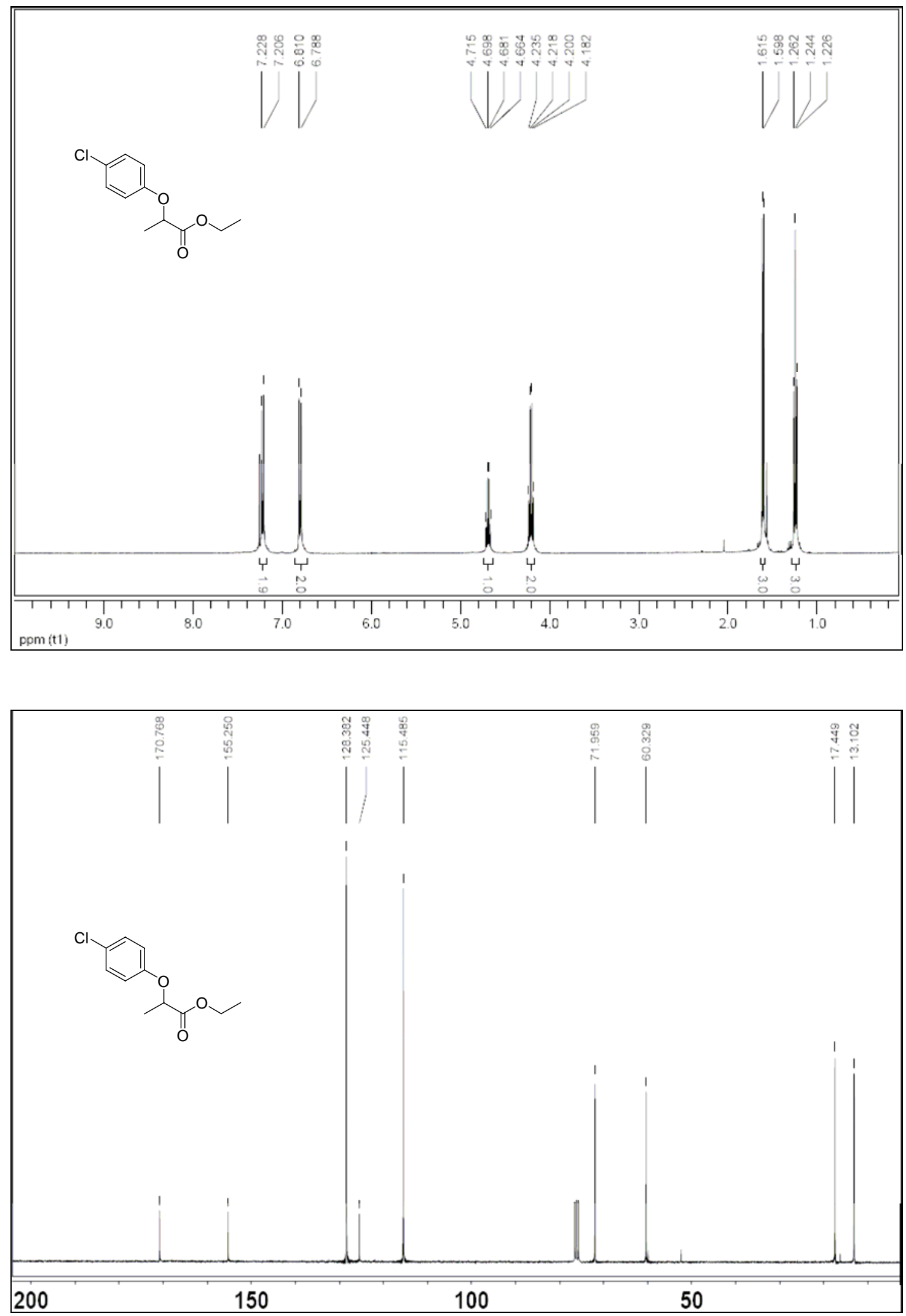
(+)-Methyl 4-(1-ethoxy-1-oxopropan-2-yloxy)benzoate(4n)
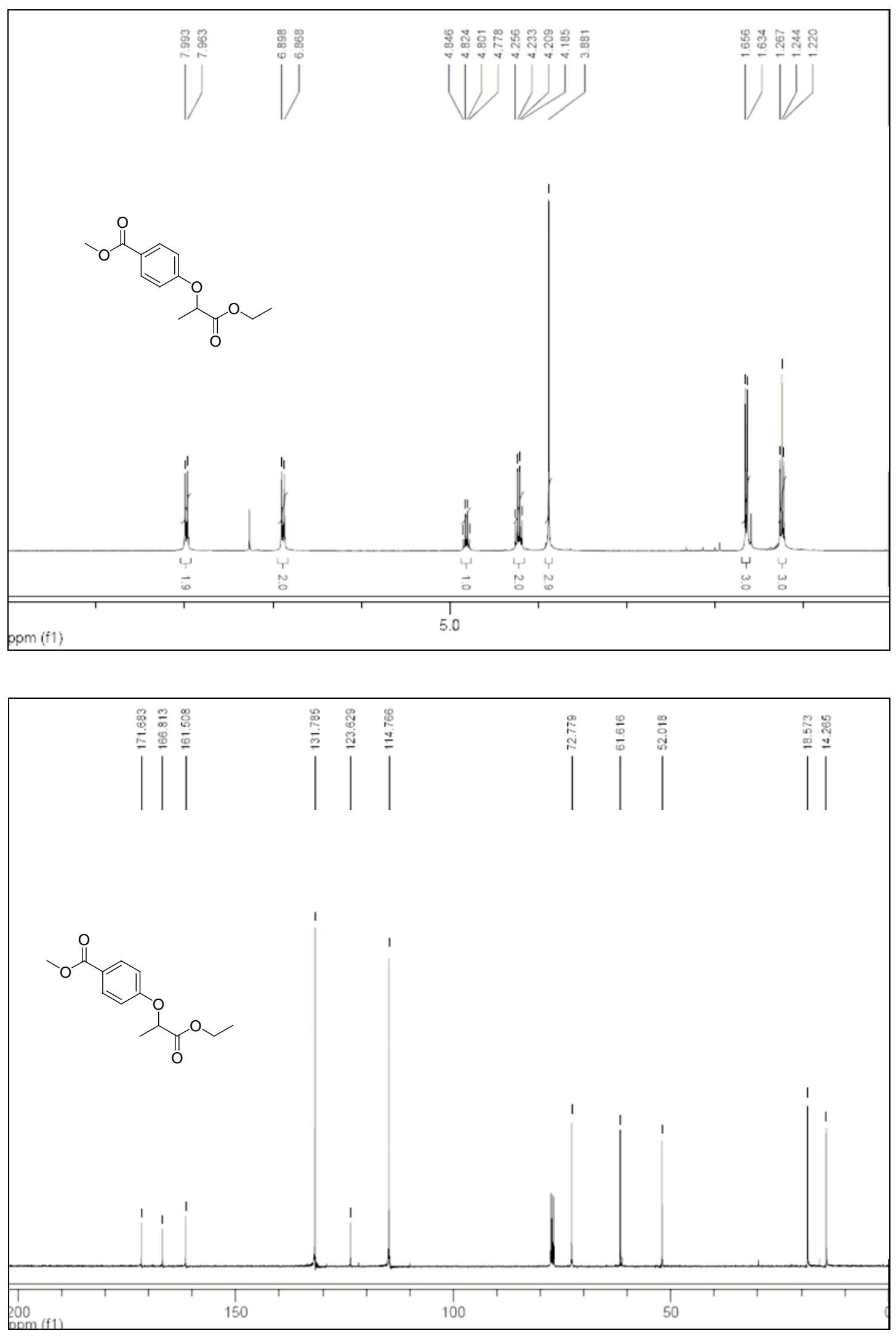

S14 
(+)-Ethyl 2-(2,4-dimethylphenoxy)propionate (40)
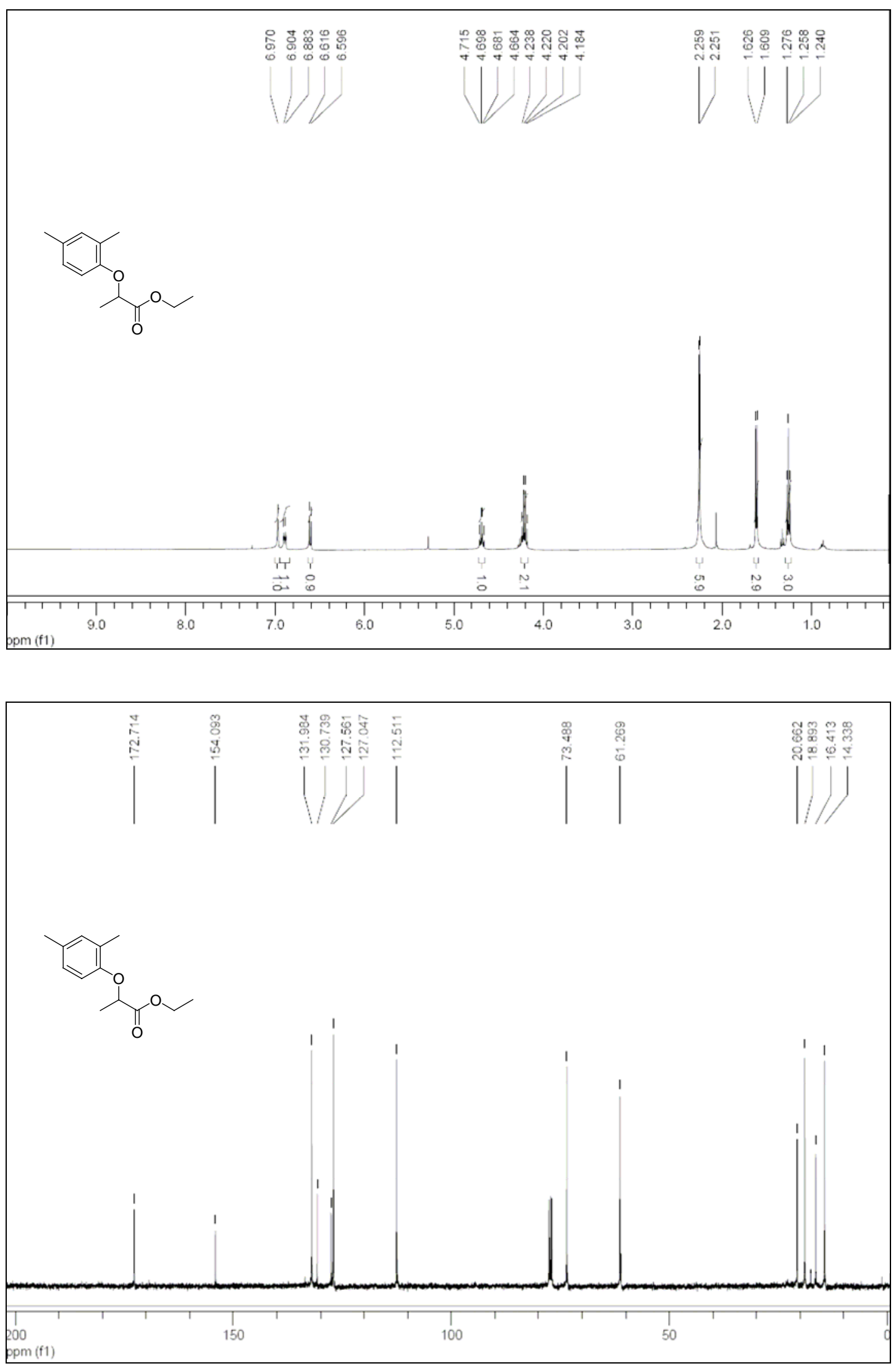
(+)-Ethyl 2-(3,5-diphenylphenoxy)propionate (4p)
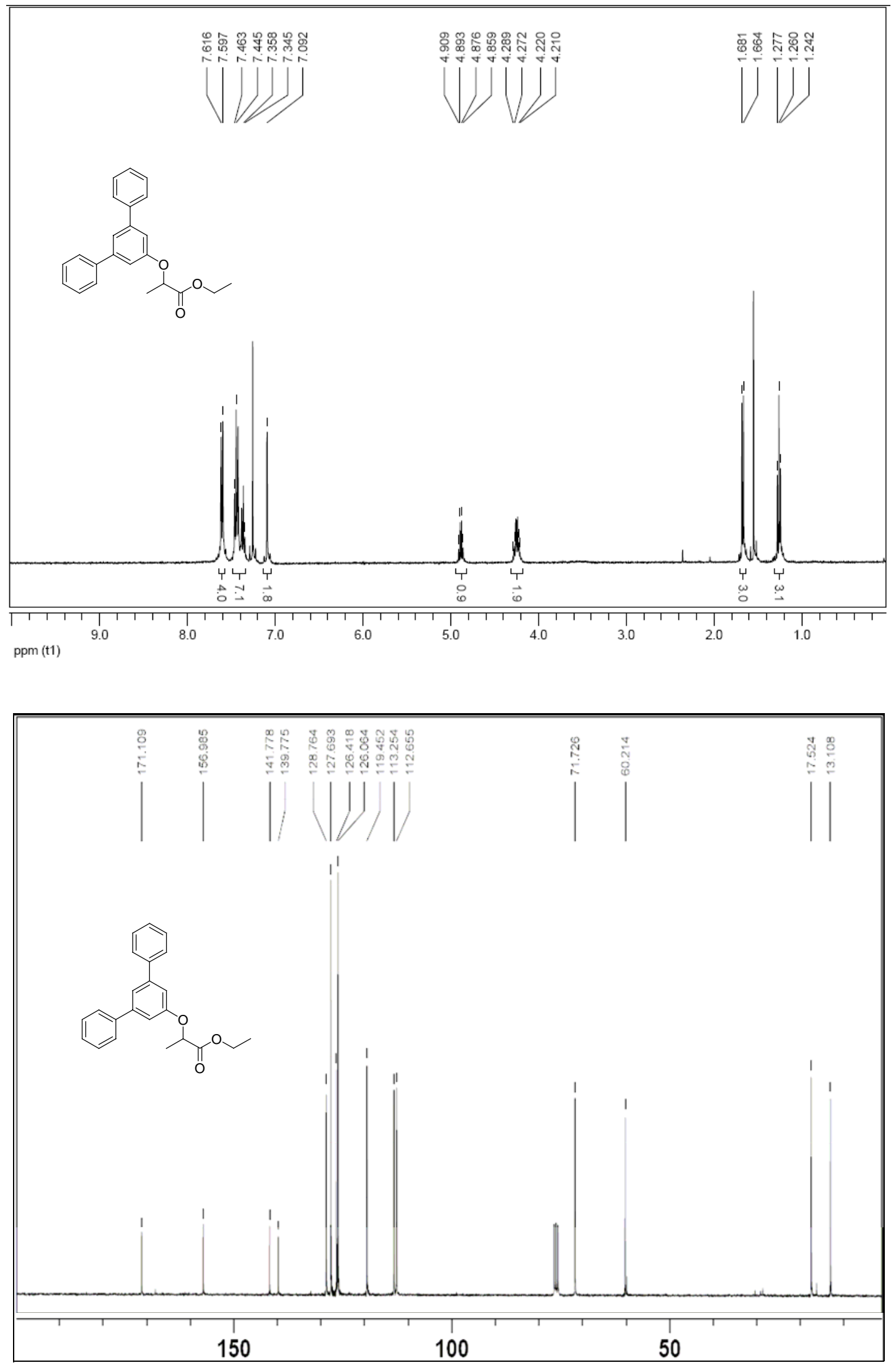
(+)-Ethyl 2-(3-phenylpropoxy)propionate (4s)
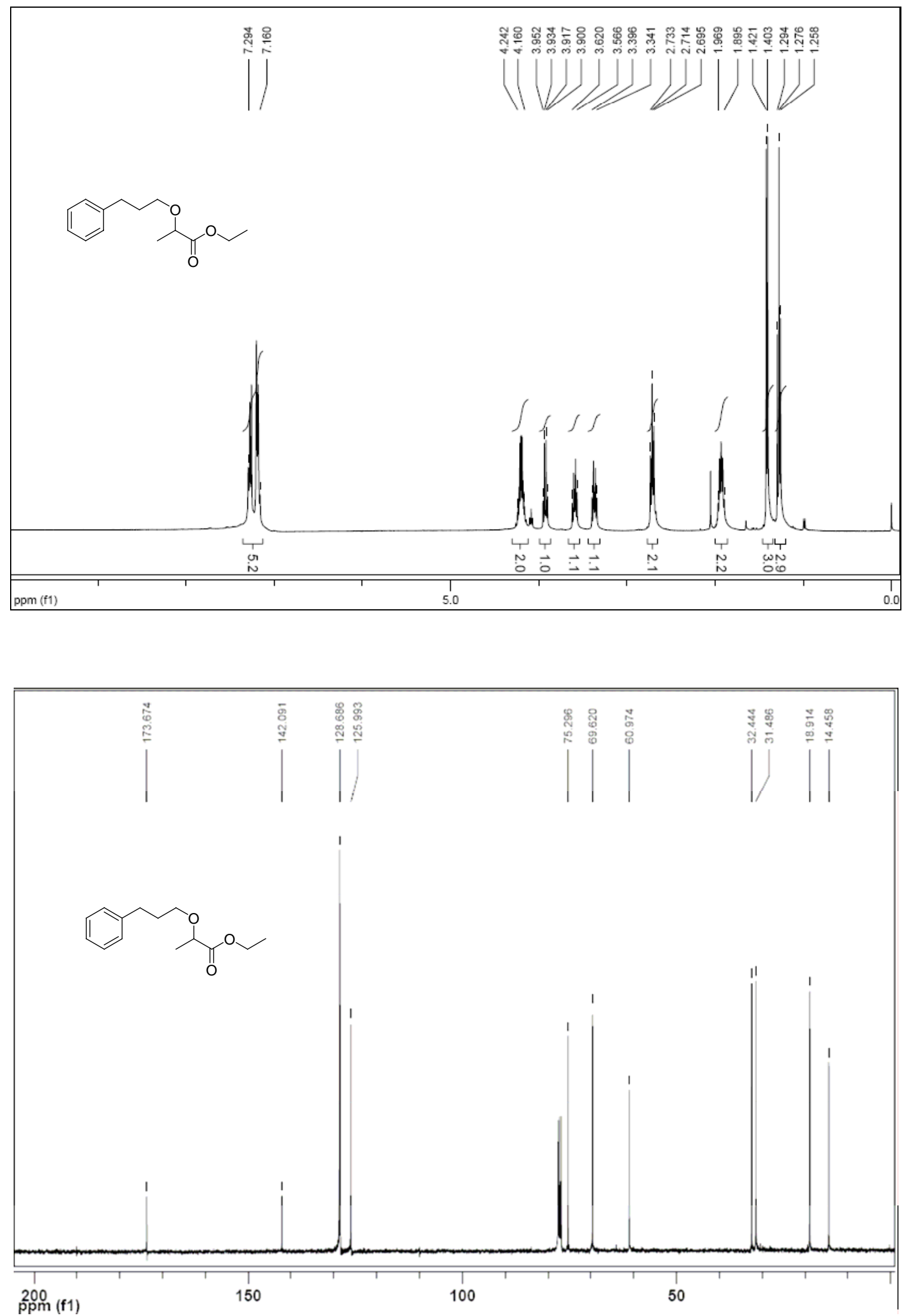
(+)-tert-Butyl 2-phenoxypropionate (4w)
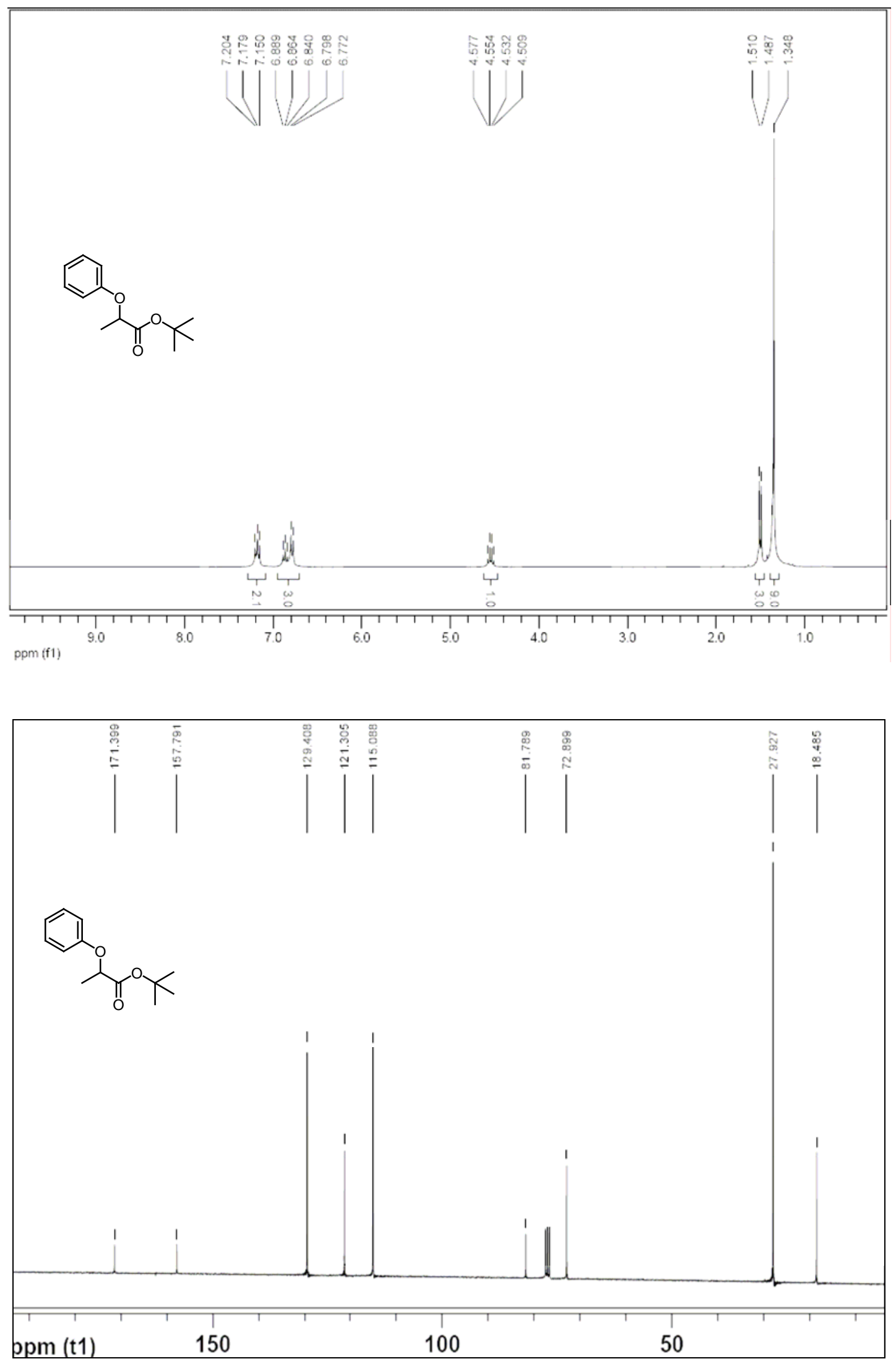

S18 
6. HPLC and SFC Charts for O-H Insertion Products

(R)-(+)-Ethyl 2-phenoxypropionate (4a)
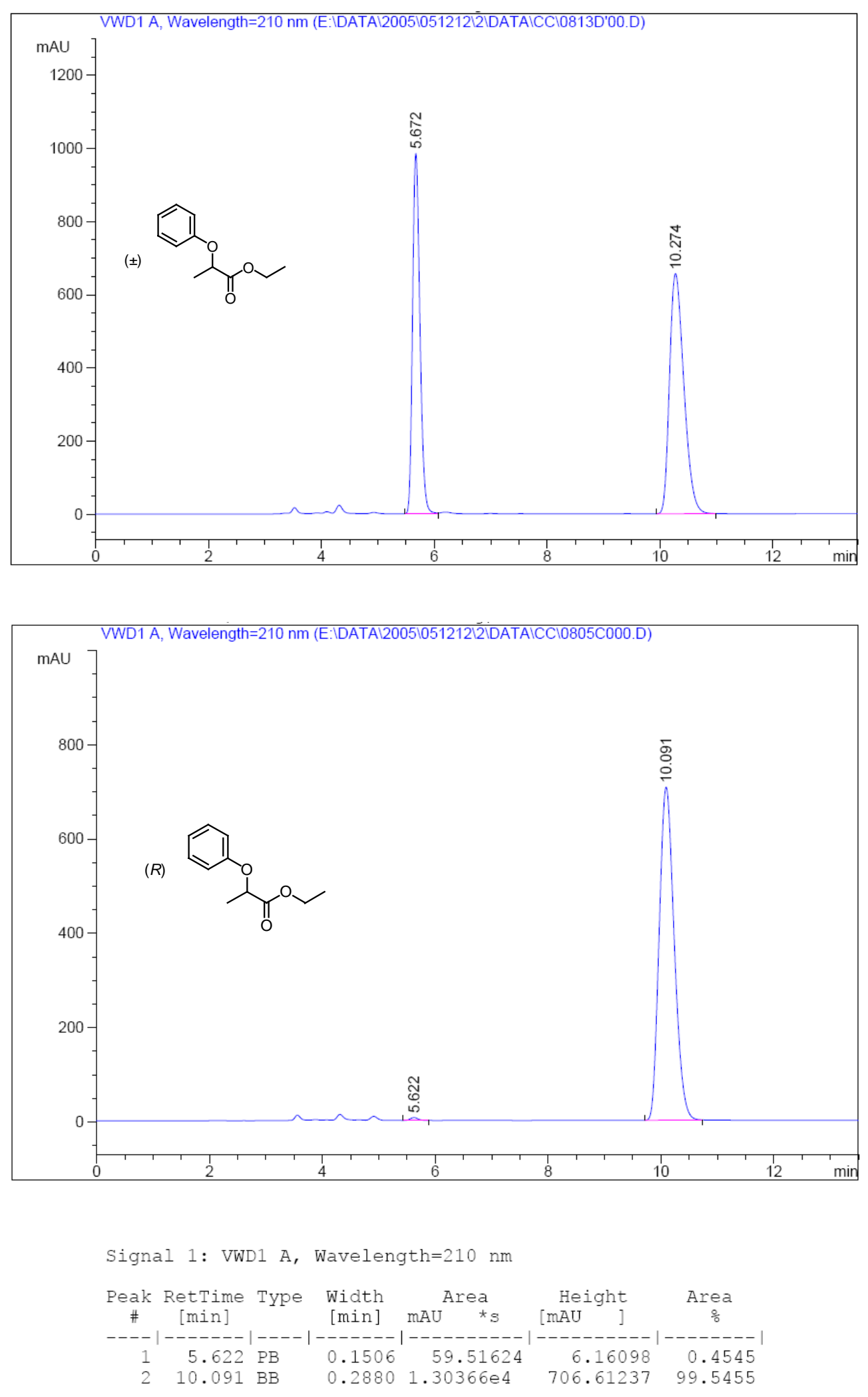

S19 
(+)-Ethyl 2-(o-tolyloxy)propionate (4b)
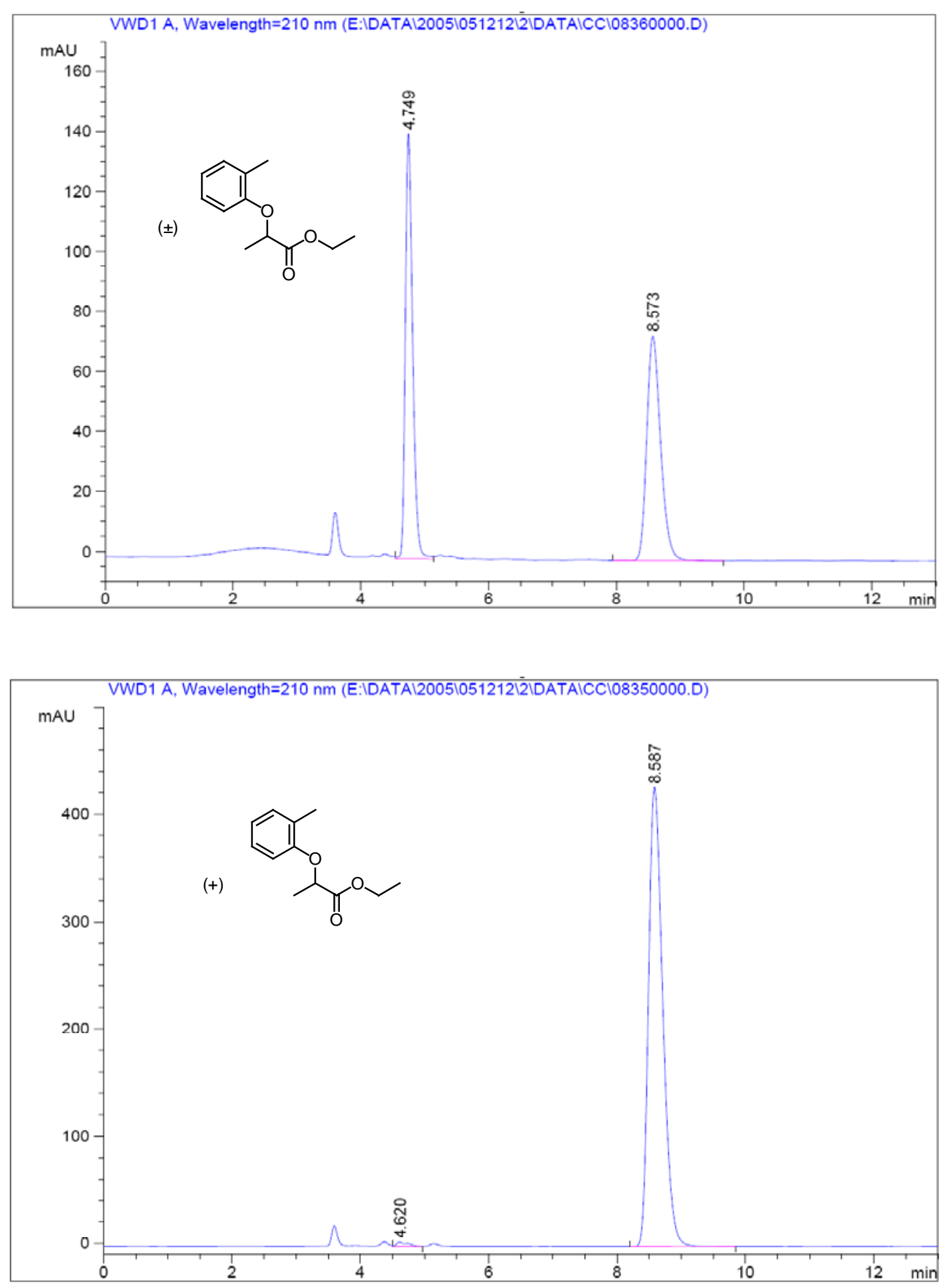

Signal 1: VWD1 A, Wavelength=210 nm

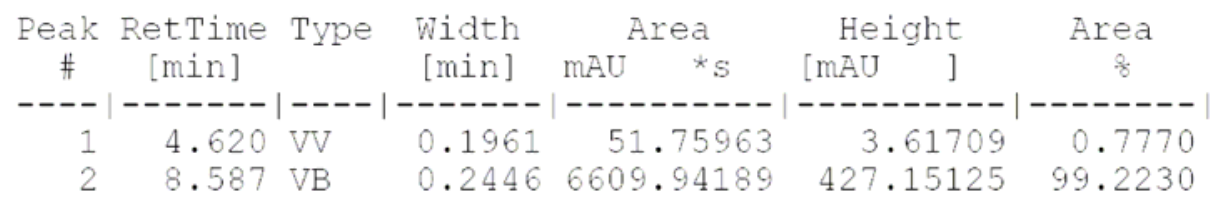

S20 
(+)-Ethyl 2-(2-methoxyphenoxy)propionate(4c)
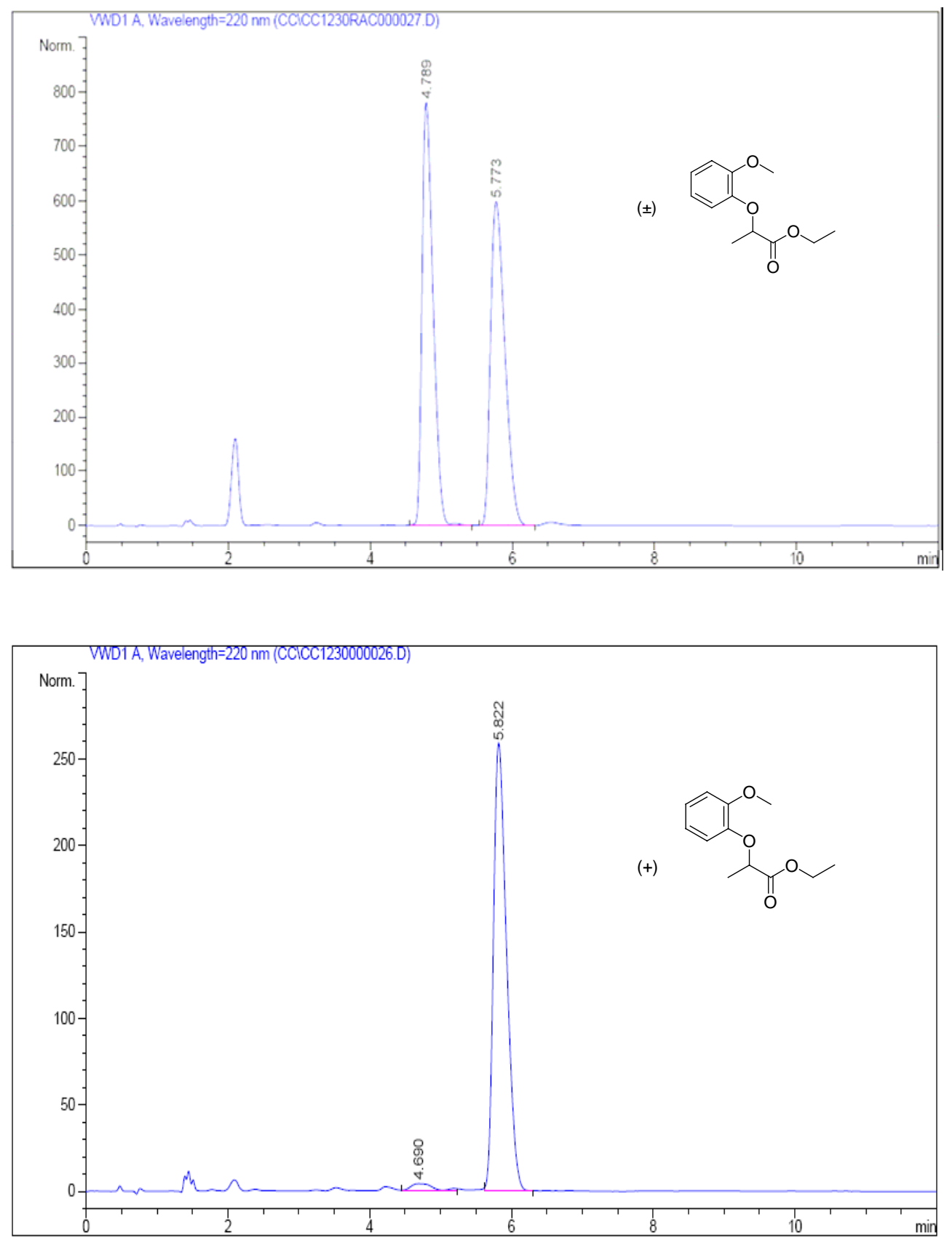

\begin{tabular}{|c|c|c|c|c|c|}
\hline Peak & RetTime & Type & width & Area & Area \\
\hline 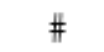 & {$[\mathrm{min}$} & & {$[\mathrm{m}$} & $\mathrm{mAU}$ & $\frac{8}{8}$ \\
\hline & & $\begin{array}{l}\mathrm{VB} \\
\mathrm{BB}\end{array}$ & & $\begin{array}{r}92.73444 \\
3318.56299\end{array}$ & 97.2815 \\
\hline
\end{tabular}




\section{(-)-Ethyl 2-(o-phenyl-phenoxy)propionate (4d)}
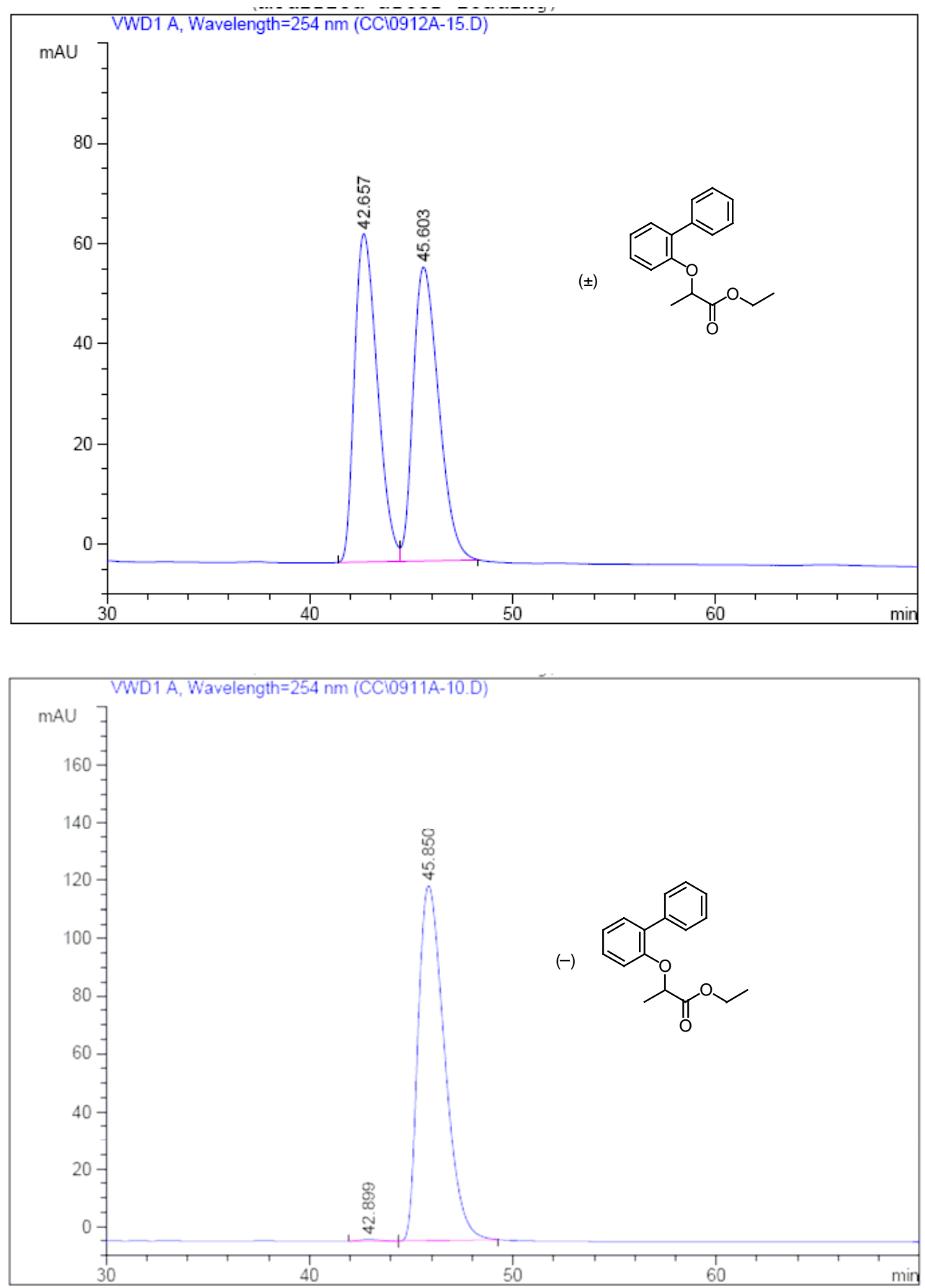

Signal 1: VWD1 A, Wavelength=254 nm

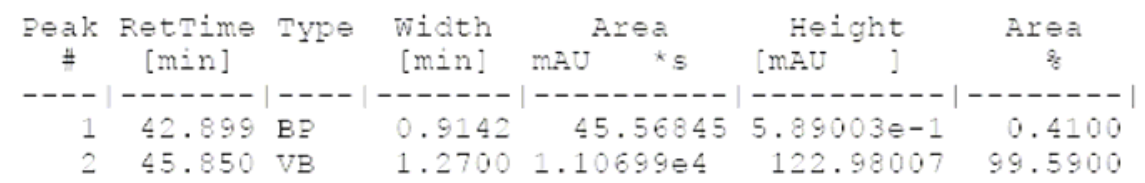


(+)-Ethyl 2-(m-tolyloxy)propionate (4e)
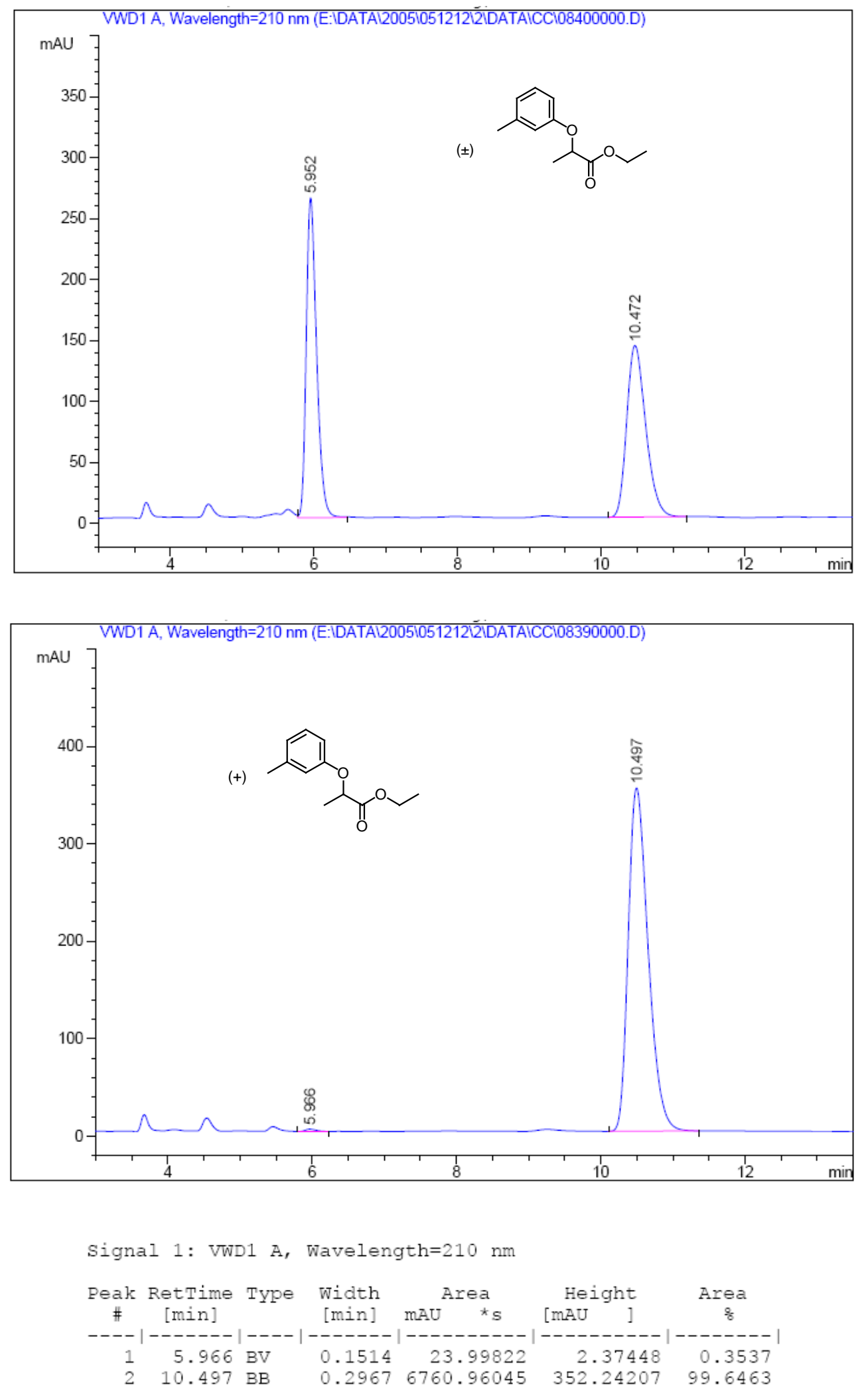

S23 
(+)-Ethyl 2-(3-methoxyphenoxy)propionate(4f)
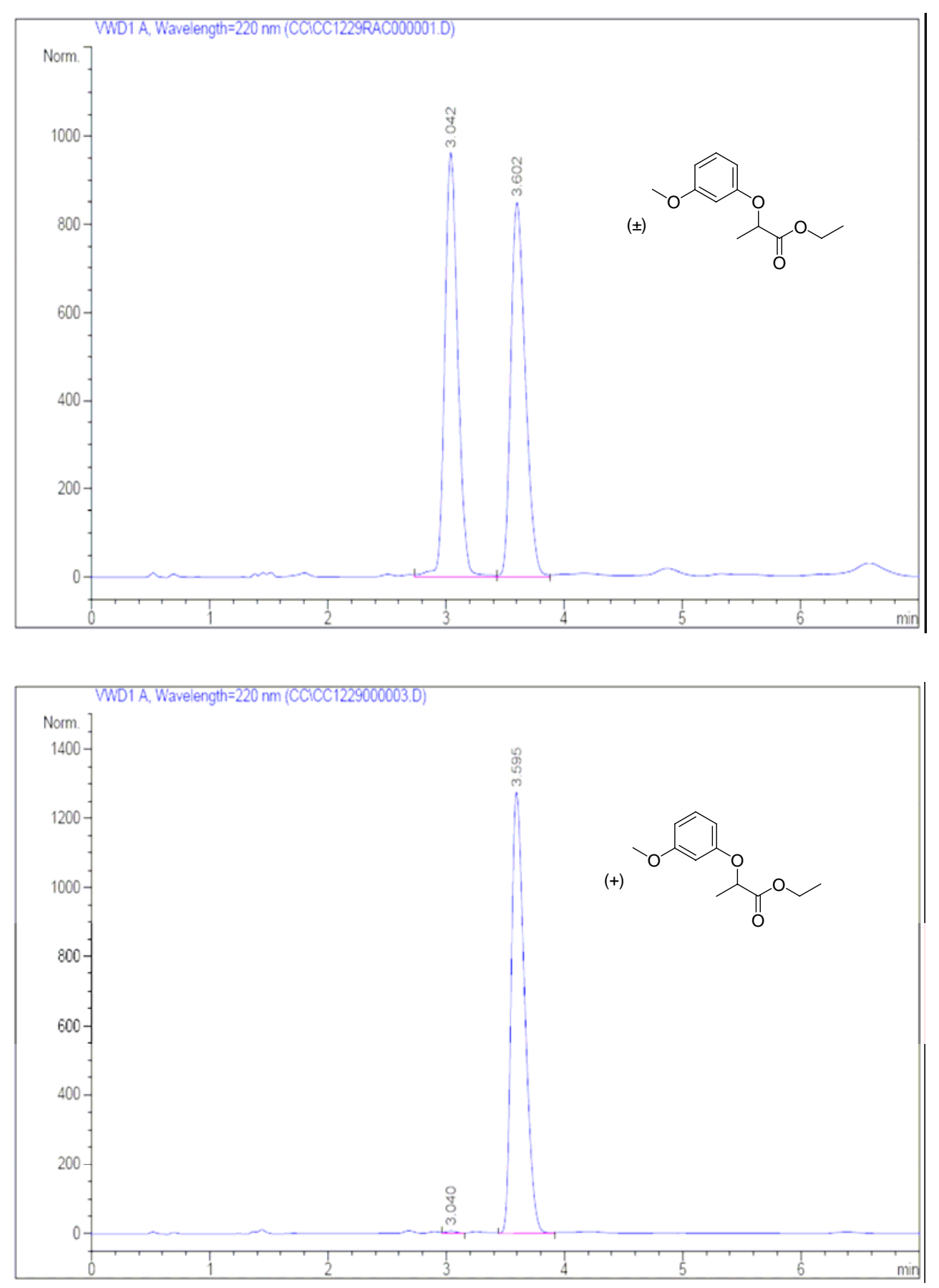

\begin{tabular}{|c|c|c|c|c|c|}
\hline Peak & RetTime & Type & width & Area & Area \\
\hline$\#$ & [min] & & {$[\min ]$} & $\mathrm{mAU} \star \star s$ & $\%$ \\
\hline $\begin{array}{l}1 \\
2\end{array}$ & $\begin{array}{l}3.040 \\
3.595\end{array}$ & $\begin{array}{l}\text { VV } \\
\text { VB }\end{array}$ & $\begin{array}{l}0.1092 \\
0.1219\end{array}$ & $\begin{array}{r}51.89931 \\
9998.22070\end{array}$ & $\begin{array}{r}0.5164 \\
99.4836\end{array}$ \\
\hline
\end{tabular}


(+)-Ethyl 2-(m-bromophenoxy)propionate (4g)
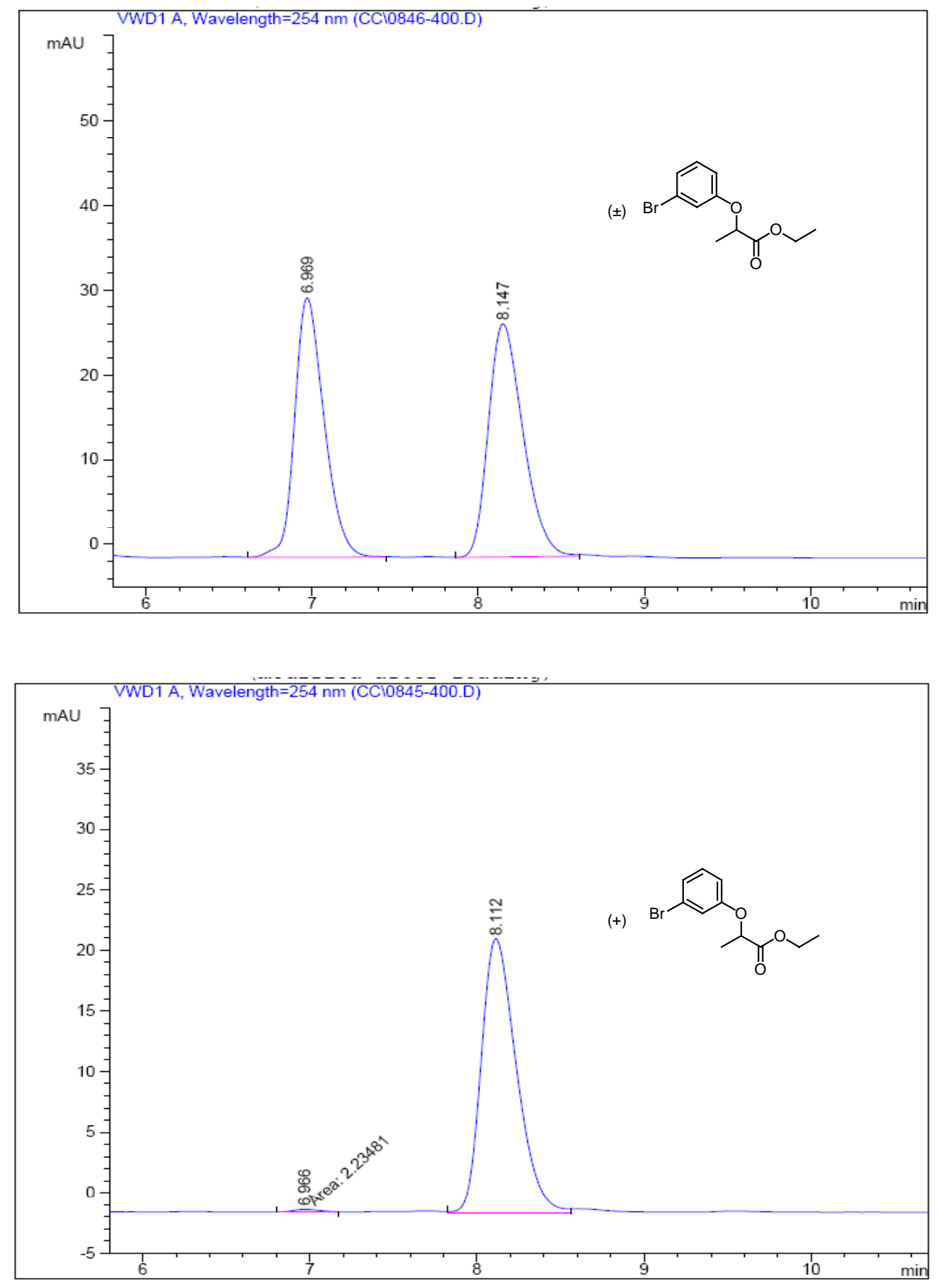

Signal 1: VWD1 A, Wavelength=254 nm

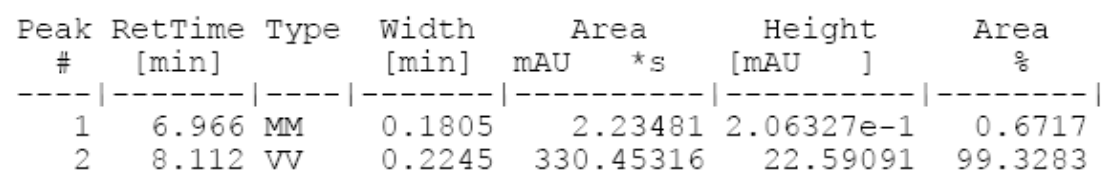

S25 
(+)-Ethyl 2-(m-phenyl-phenoxy)propionate (4h)
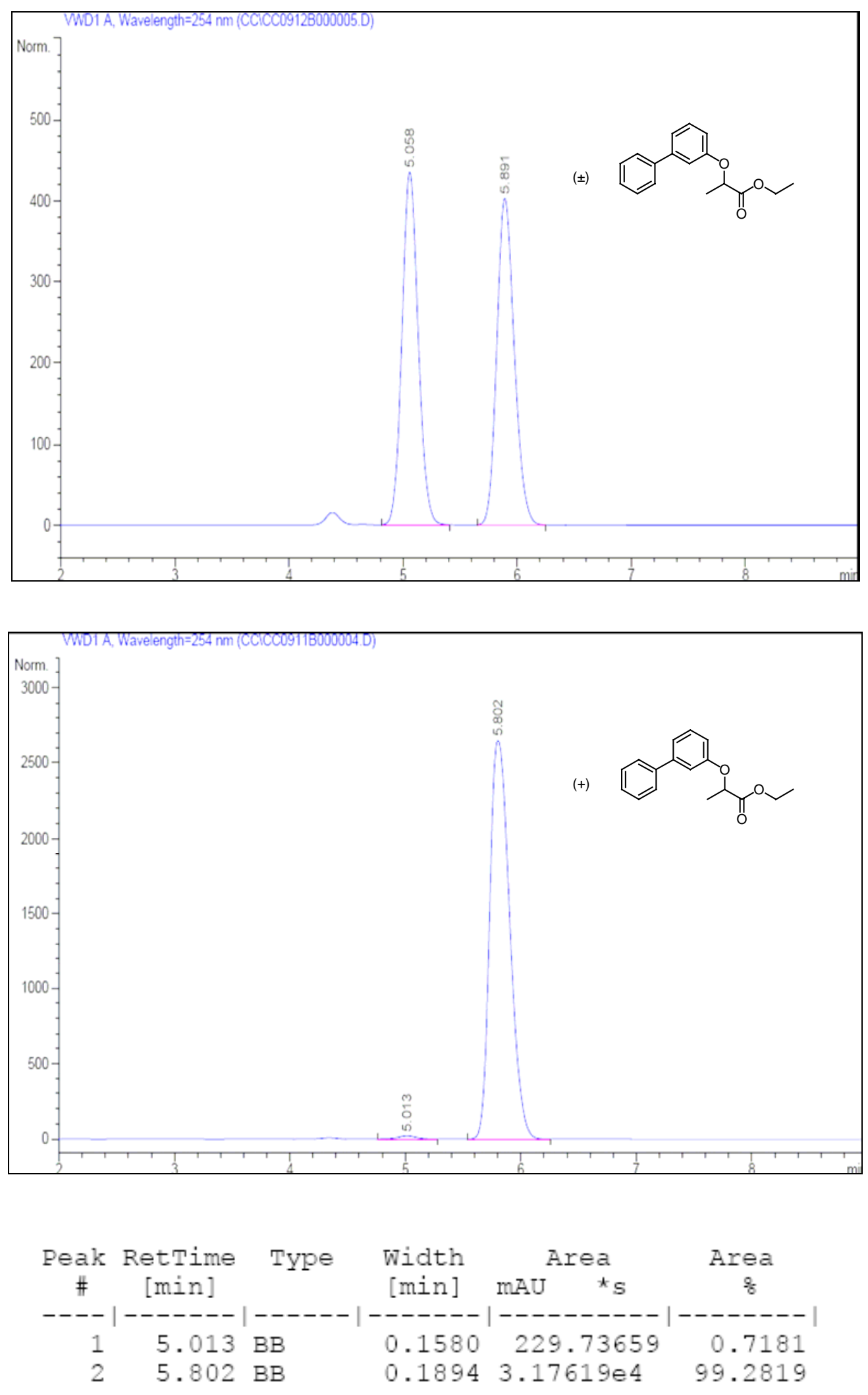

S26 
(+)-Ethyl 2-(p-tolyloxy)propionate (4i)
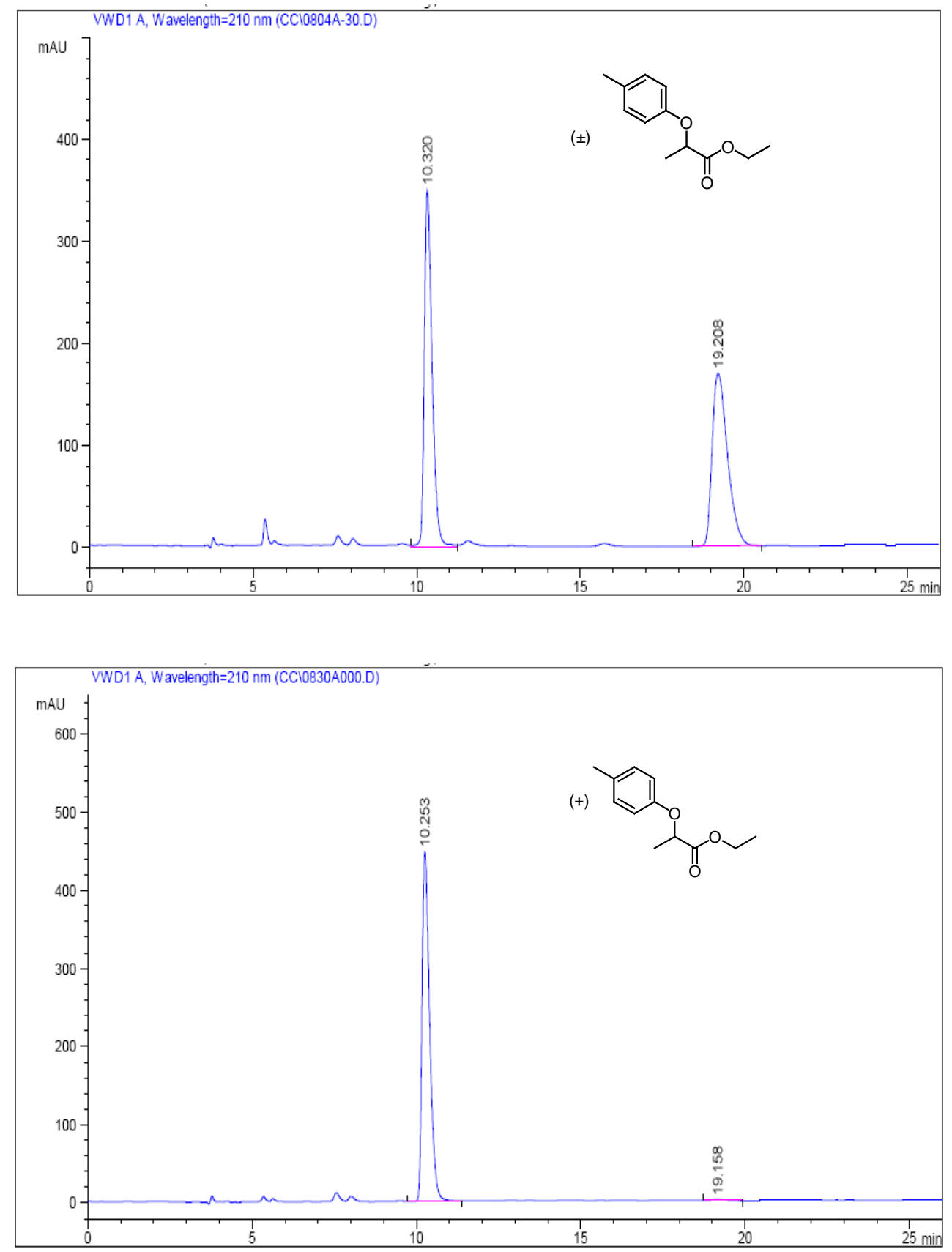

Signal 1: VWD1 A, Wavelength=210 nm

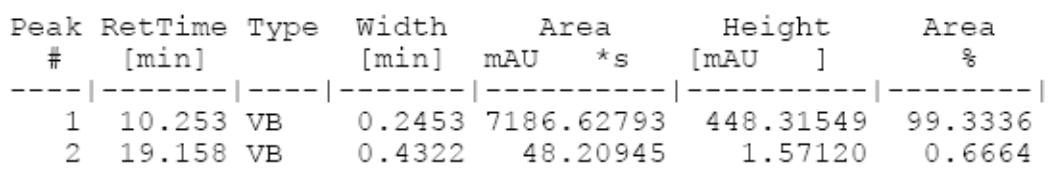

S27 
(+)-Ethyl 2-(3-methoxyphenoxy)propionate(4j)
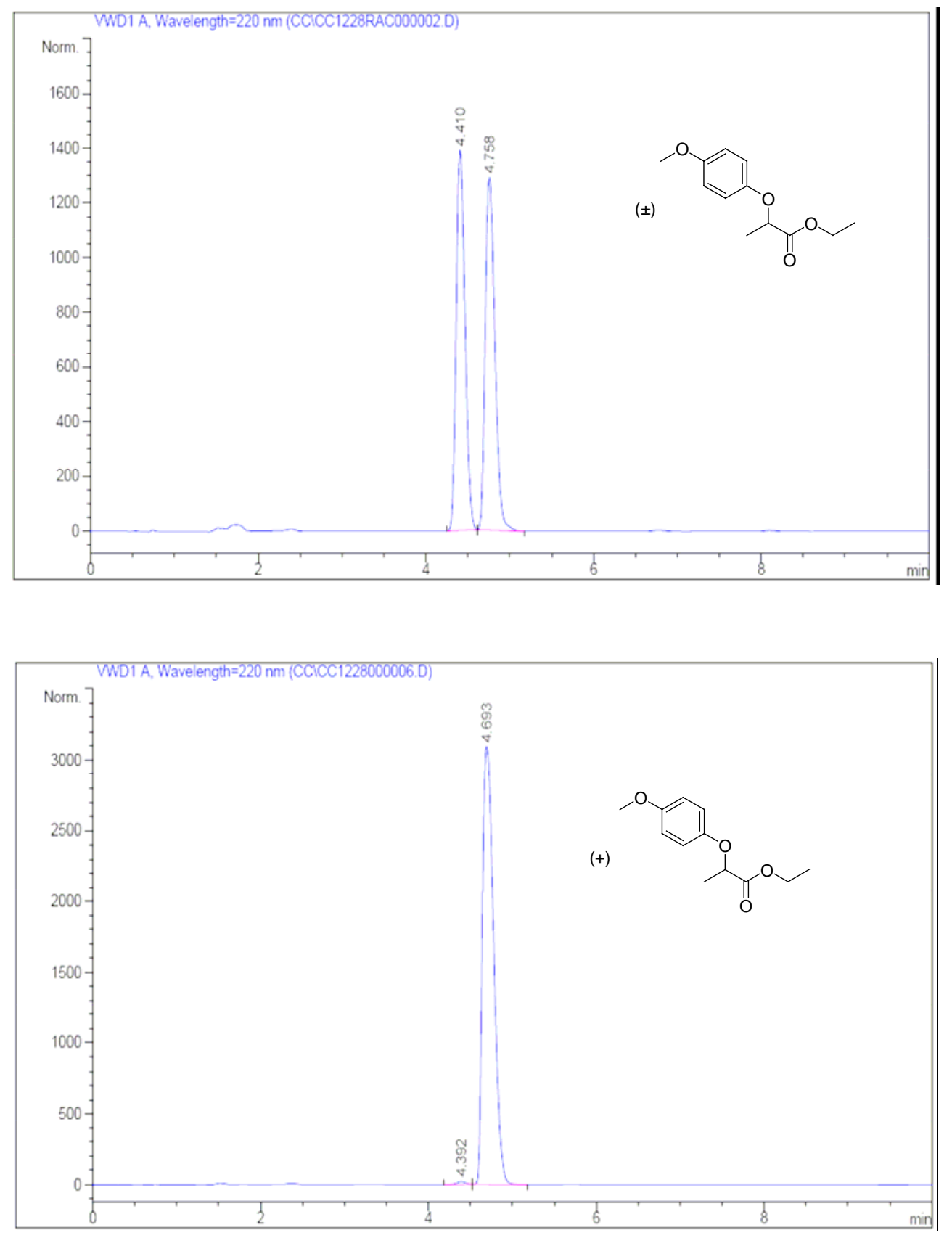

\begin{tabular}{|c|c|c|c|c|c|}
\hline Peak & Retrime & Type & width & Area & Area \\
\hline$\#$ & [min] & & [min] & $\mathrm{mZU}$ & sक \\
\hline & 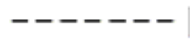 & & ------- & | ---------- & ----ー-ー- \\
\hline 1 & $\leq .392$ & $B B$ & 0.1124 & 144.40123 & 0.5009 \\
\hline 2 & $\leq .693$ & $B B$ & 0.1489 & $2.86832 e 4$ & 99.4991 \\
\hline
\end{tabular}


(+)-Ethyl 2-(p-tert-butylphenoxy)propionate (4k)
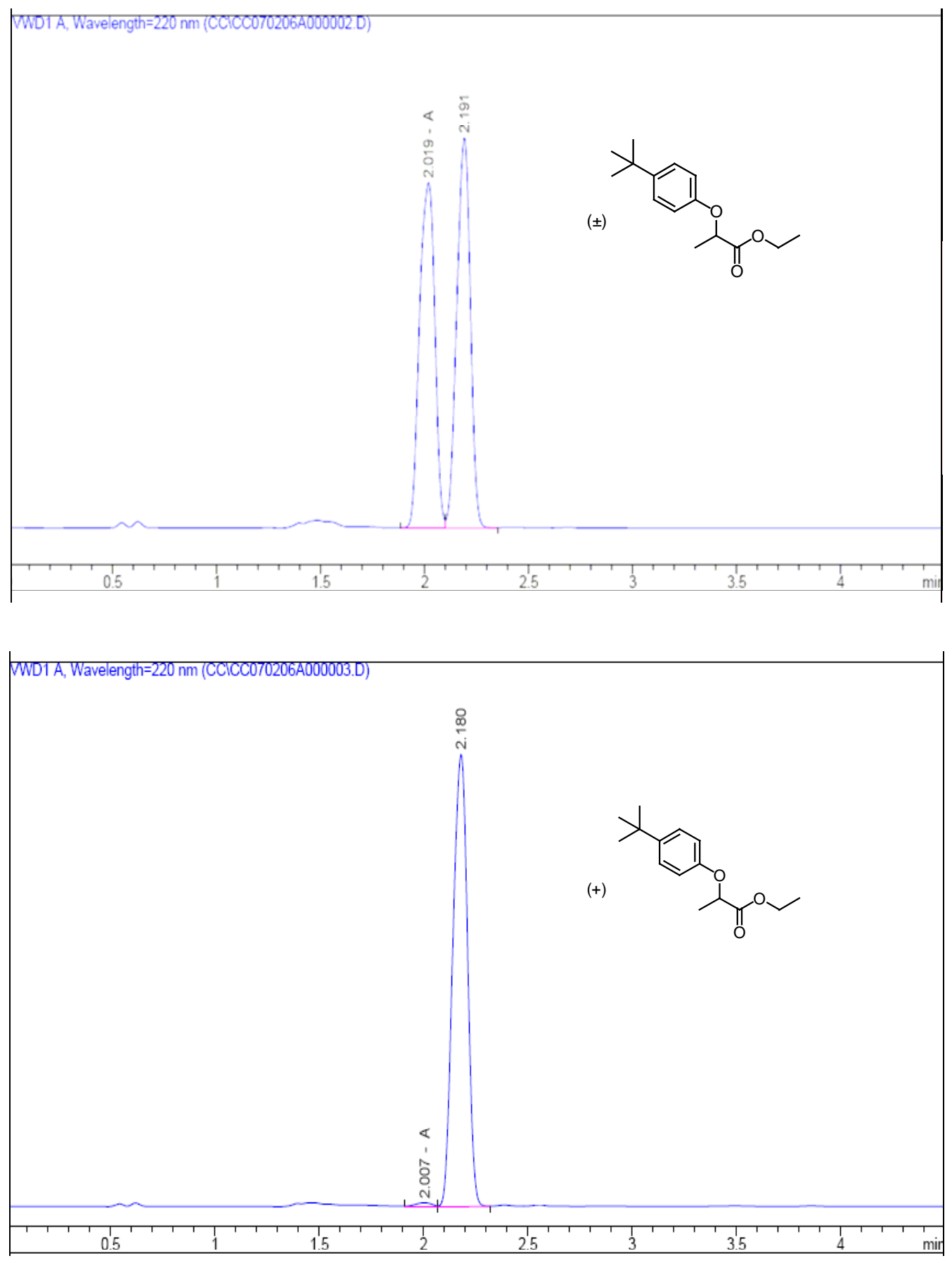

Signal 1: VWD1 A, Wavelength=220 nm

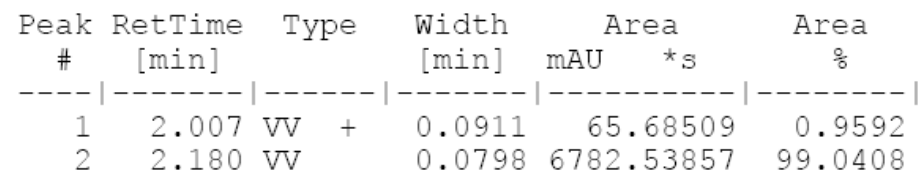


(R)-(+)-Ethyl 2-(p-chlorophenoxy)propionate (4l)
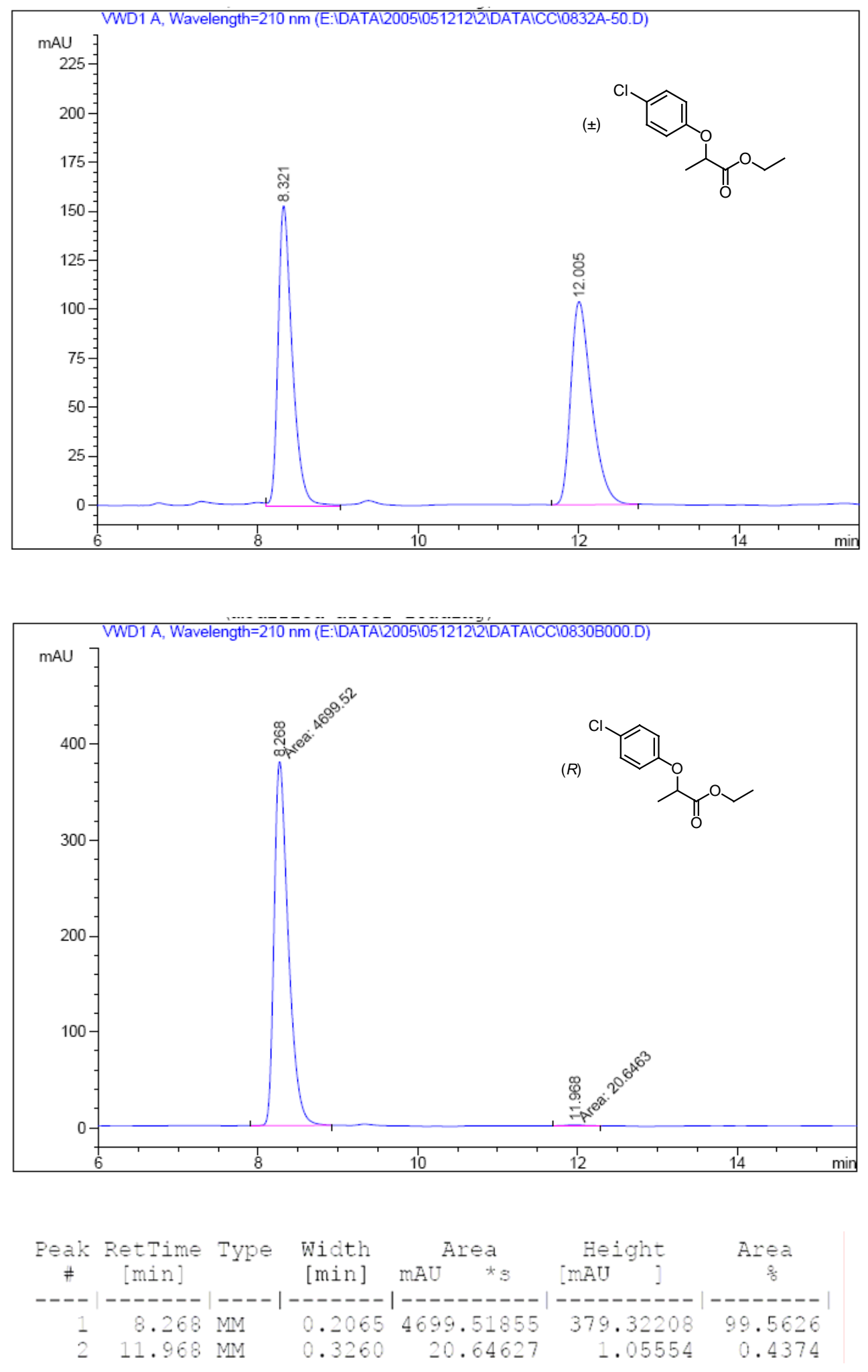

S30 
(+)-Ethyl 2-(3,5-difluorophenoxy)propionate (4m)
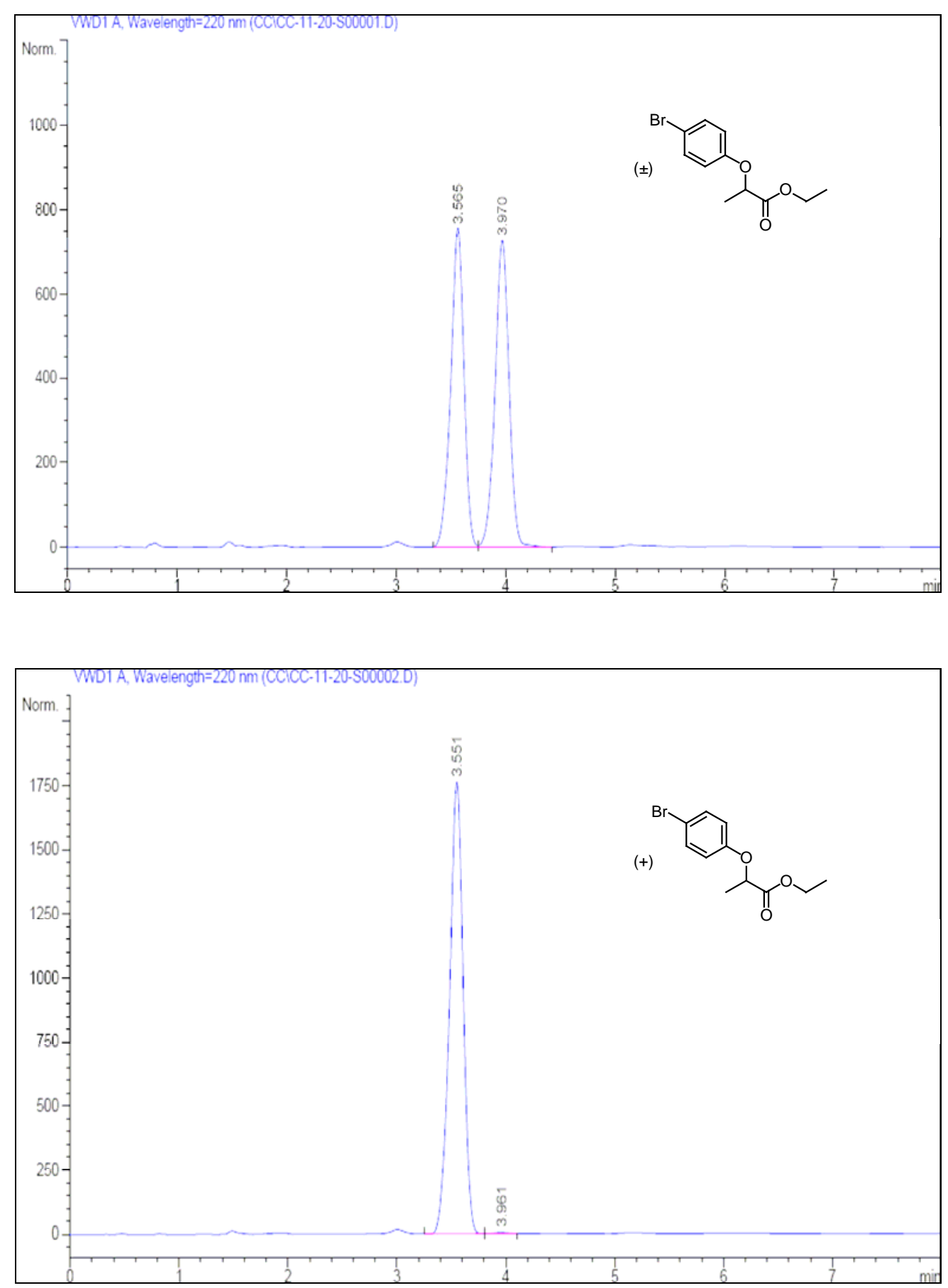

\begin{tabular}{|c|c|c|c|c|c|}
\hline Peak & Retrime & Type & width & Area & Area \\
\hline$=$ & [min] & & [min] & $\mathrm{mAU} * \mathrm{~s}$ & \\
\hline$\frac{1}{2}$ & $\begin{array}{l}3.551 \\
3.961\end{array}$ & $\begin{array}{l}\mathrm{BB} \\
\mathrm{BB}\end{array}$ & $\begin{array}{l}0.1317 \\
0.1139\end{array}$ & $\begin{array}{c}1.52898 \mathrm{e} 4 \\
28.50722\end{array}$ & $\begin{array}{r}99.8139 \\
0.1861\end{array}$ \\
\hline
\end{tabular}


(+)-Methyl 4-(1-ethoxy-1-oxopropan-2-yloxy)benzoate (4n)
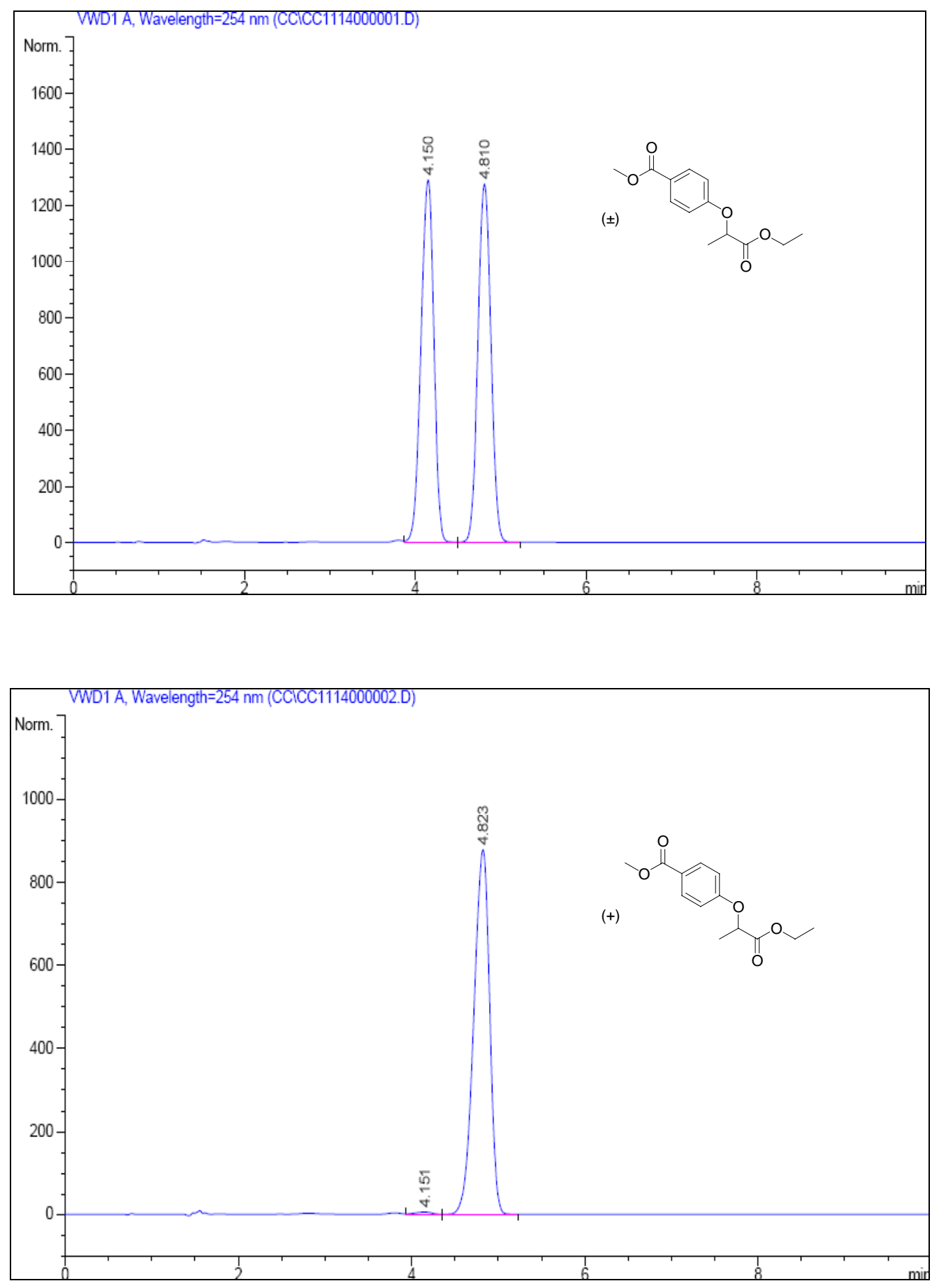

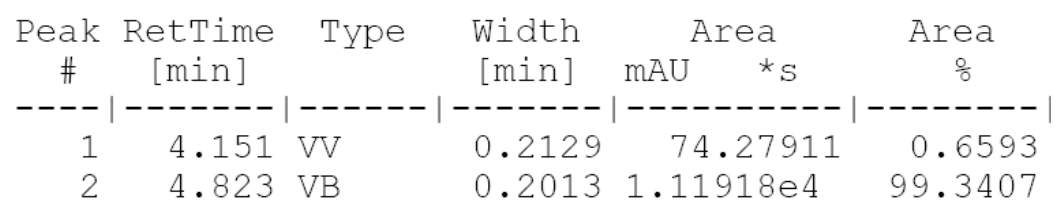


(+)-Ethyl 2-(2,4-dimethylphenoxy)propionate (40)
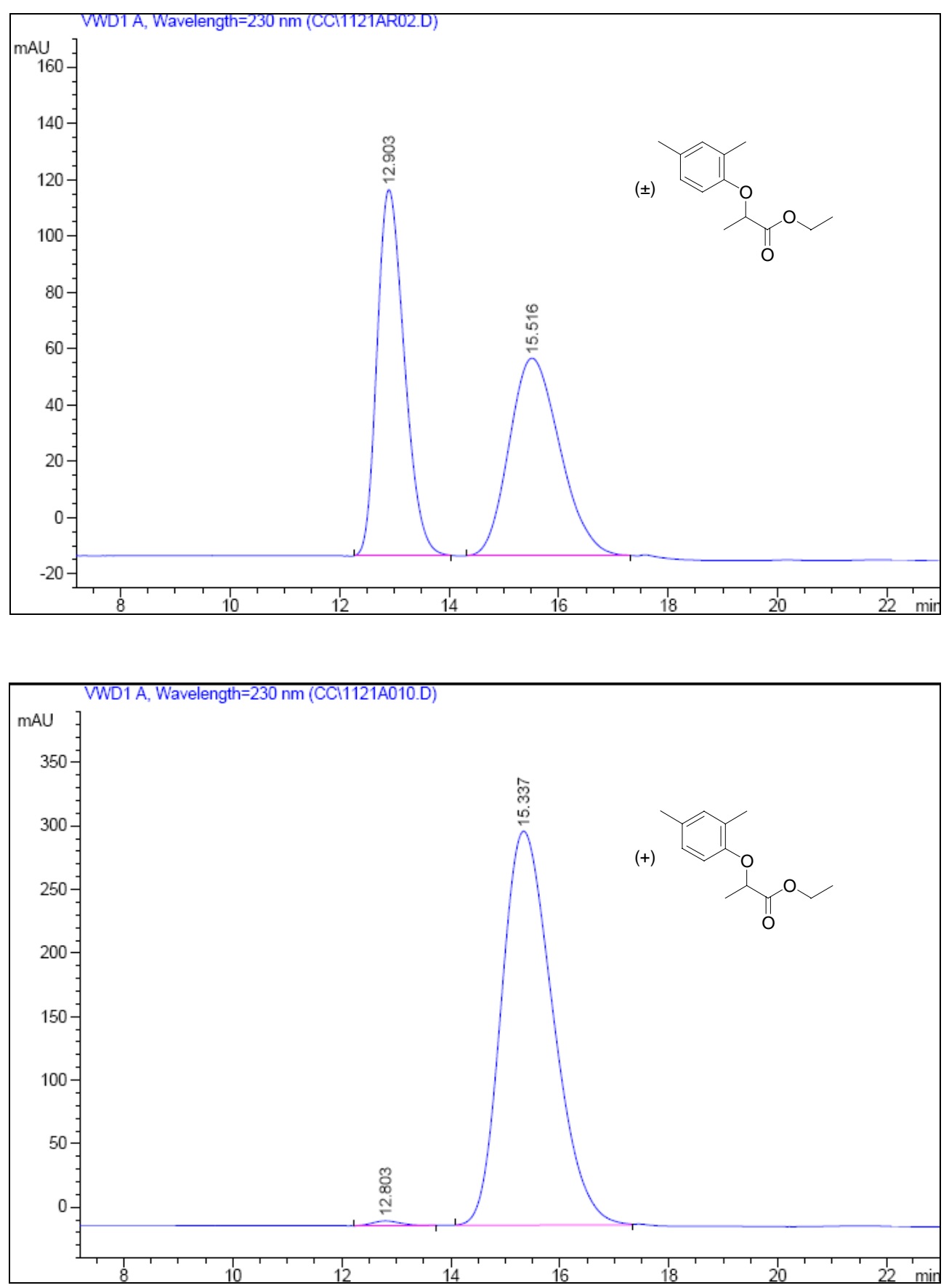

\begin{tabular}{|c|c|c|c|c|c|c|}
\hline Peak & RetTime & Type & width & Area & Height & Area \\
\hline$\#$ & [min] & & [min] & $\mathrm{mAU}$ & {$[\mathrm{mAU}$} & \\
\hline 1 & $\begin{array}{l}12.803 \\
15.337\end{array}$ & $\begin{array}{l}\mathrm{PB} \\
\mathrm{BB}\end{array}$ & $\begin{array}{l}0.5306 \\
1.0173\end{array}$ & $\begin{array}{c}132.84798 \\
2.03026 \mathrm{e} 4\end{array}$ & $\begin{array}{r}3.71653 \\
310.03354\end{array}$ & $\begin{array}{l}501 \\
499\end{array}$ \\
\hline
\end{tabular}


(+)-Ethyl 2-(3,5-diphenyl-phenoxy)propionate (4p)
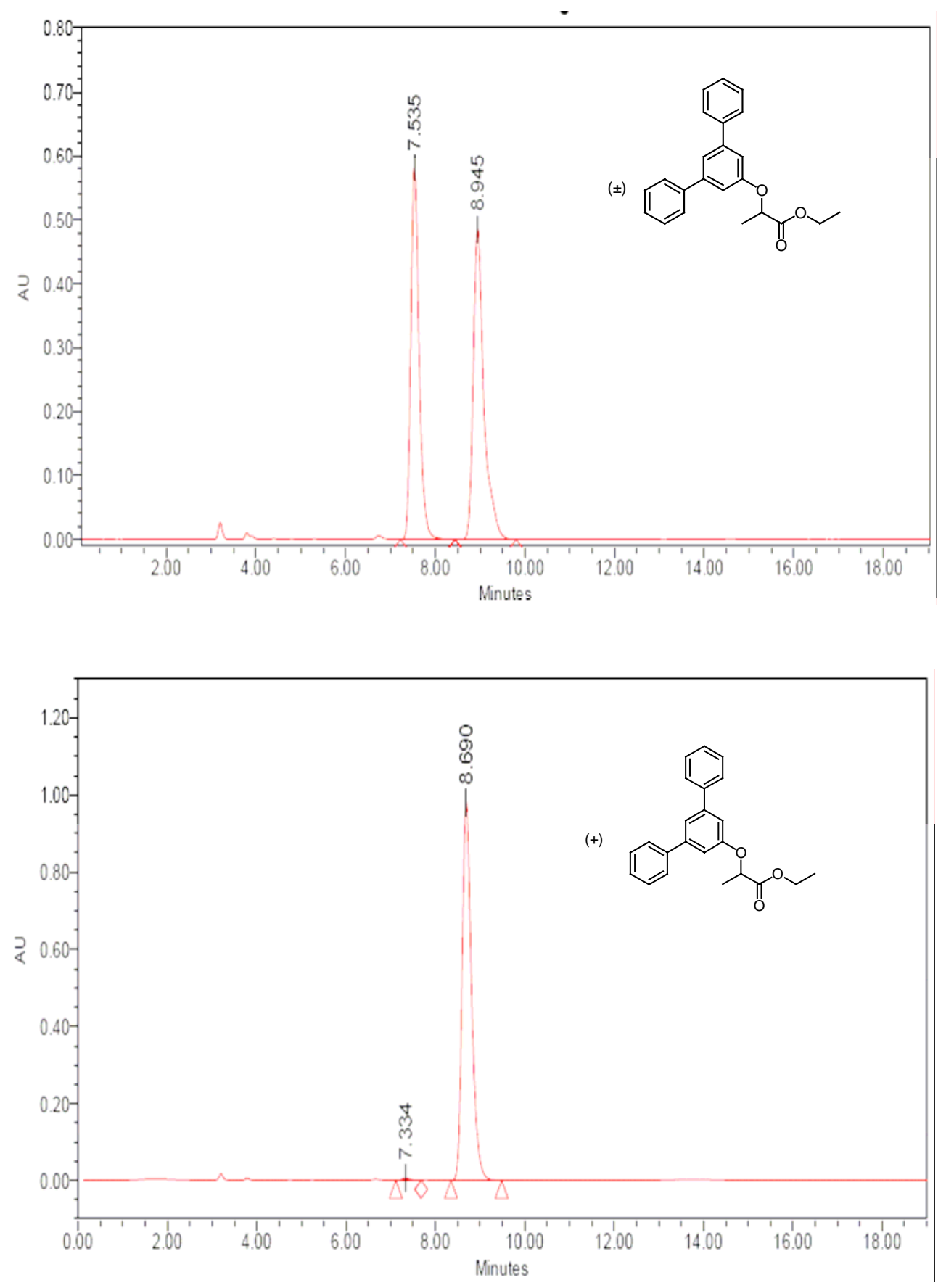

Acquired By: System

Date Acquired: $\quad$ 12/13/06 10:02:09

Acq. Method Set: $\quad c c 411$

Date Processed: $\quad$ 12/13/06 10:24:20

Processing Method $\mathrm{CC}$

Channel Name: Wvin Ch1

Proc. Chnl. Descr.: PDA $254.0 \mathrm{~nm}$

Peak Results
\begin{tabular}{|l|c|c|r|r|}
\hline & RT & Area & Height & $\%$ Area \\
\hline 1 & 7.334 & 66903 & 5254 & 0.47 \\
\hline 2 & 8.690 & 14312382 & 983246 & 99.53 \\
\hline
\end{tabular}


(R)-(-)-Ethyl 2-(naphthalen-1-yloxy)propionate (4q)
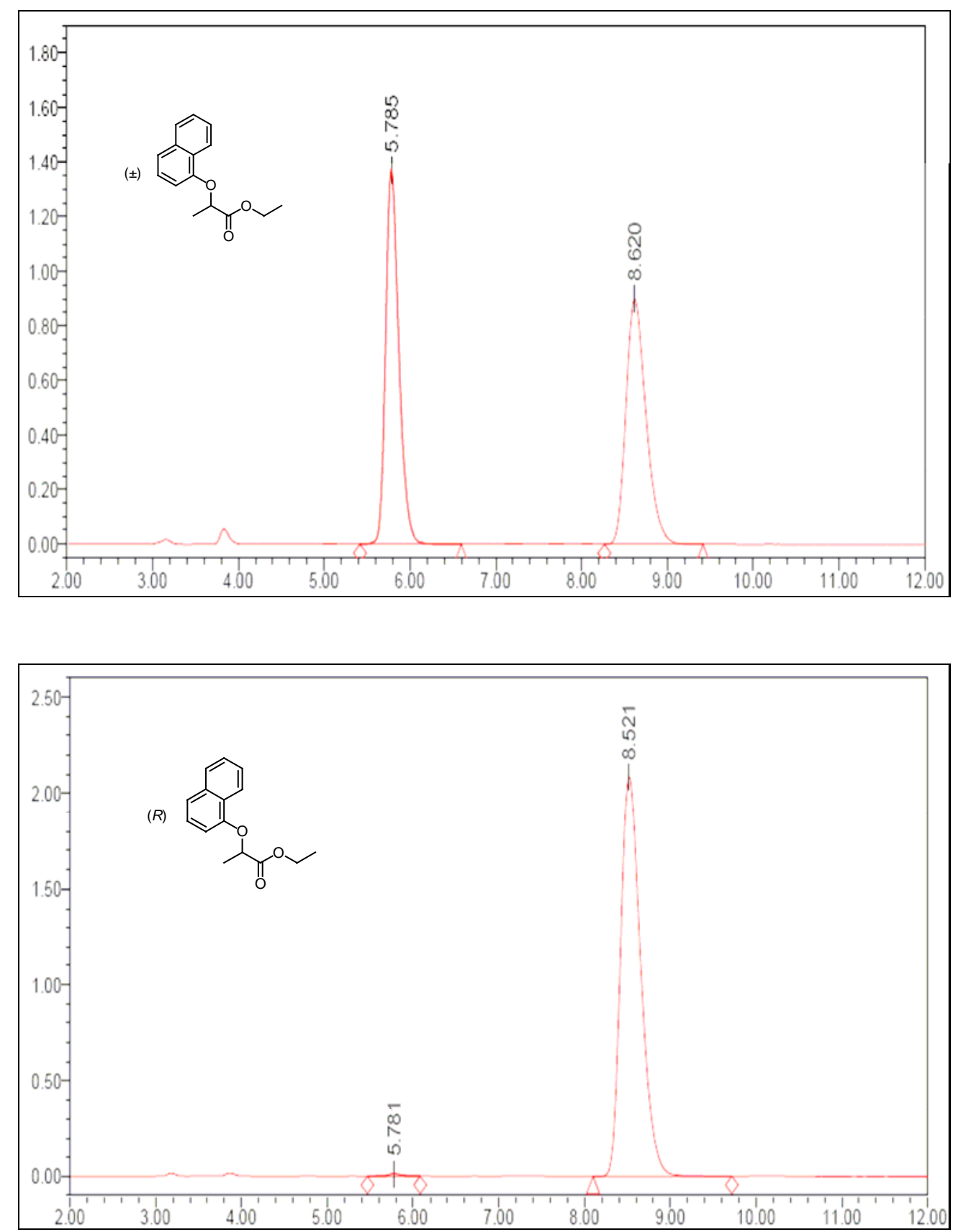

\begin{tabular}{|c|c|c|c|c|c|c|}
\hline Acquired By: & System & \multicolumn{5}{|c|}{ Peak Results } \\
\hline $\begin{array}{l}\text { Date Acquired: } \\
\text { Acq. Method Set: }\end{array}$ & $\begin{array}{l}\text { 11/24/06 21:48:14 } \\
\text { cc411 }\end{array}$ & & RT & Area & Height & $\%$ Area \\
\hline $\begin{array}{l}\text { Date Processed: } \\
\text { Processing Method }\end{array}$ & $\begin{array}{l}\text { 11/24/06 22:16:55 } \\
\text { cc0847a }\end{array}$ & 1 & 5.781 & 156577 & 12866 & 0.45 \\
\hline $\begin{array}{l}\text { Channel Name: } \\
\text { Proc Chnl Descr. }\end{array}$ & $\begin{array}{l}\text { Wvln Ch1 } \\
\text { PDA 210 } 0 \mathrm{~nm}\end{array}$ & 2 & 8.521 & 34288058 & 2088641 & 99.55 \\
\hline
\end{tabular}


(R)-(+)-Ethyl 2-(naphthalen-2-yloxy)propionate (4r)
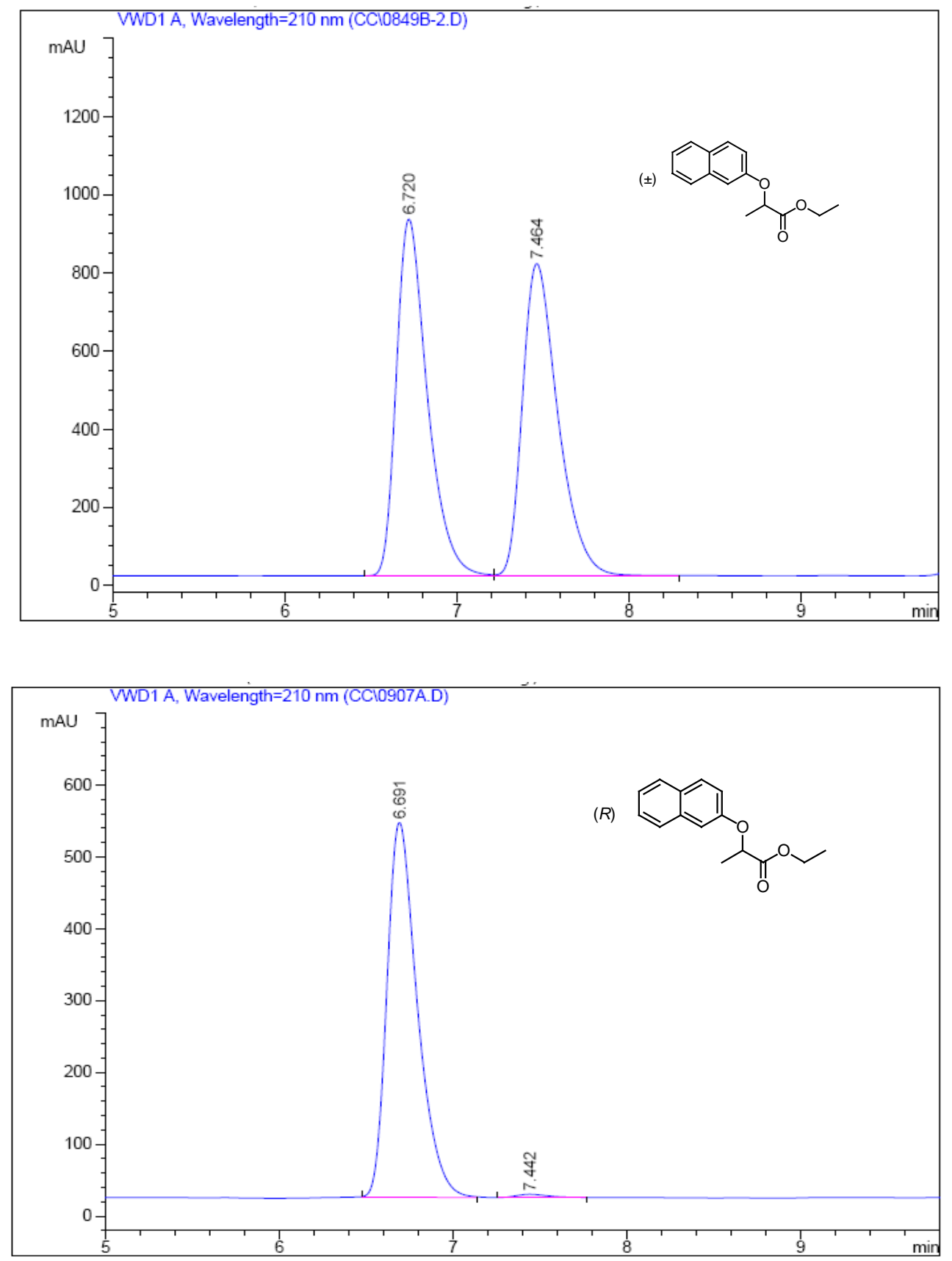

Signal 1: VWD1 A, Wavelength=210 nm

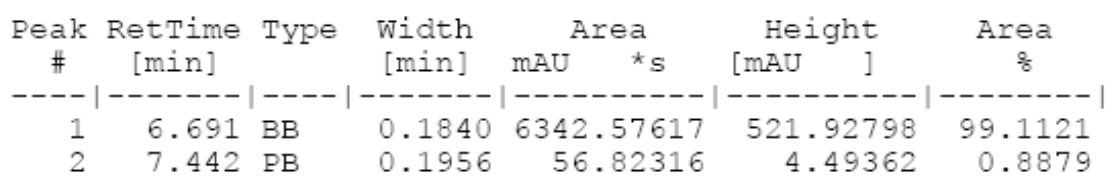

S36 
(+)-Ethyl 2-(3-phenylpropoxy)propionate (4s)
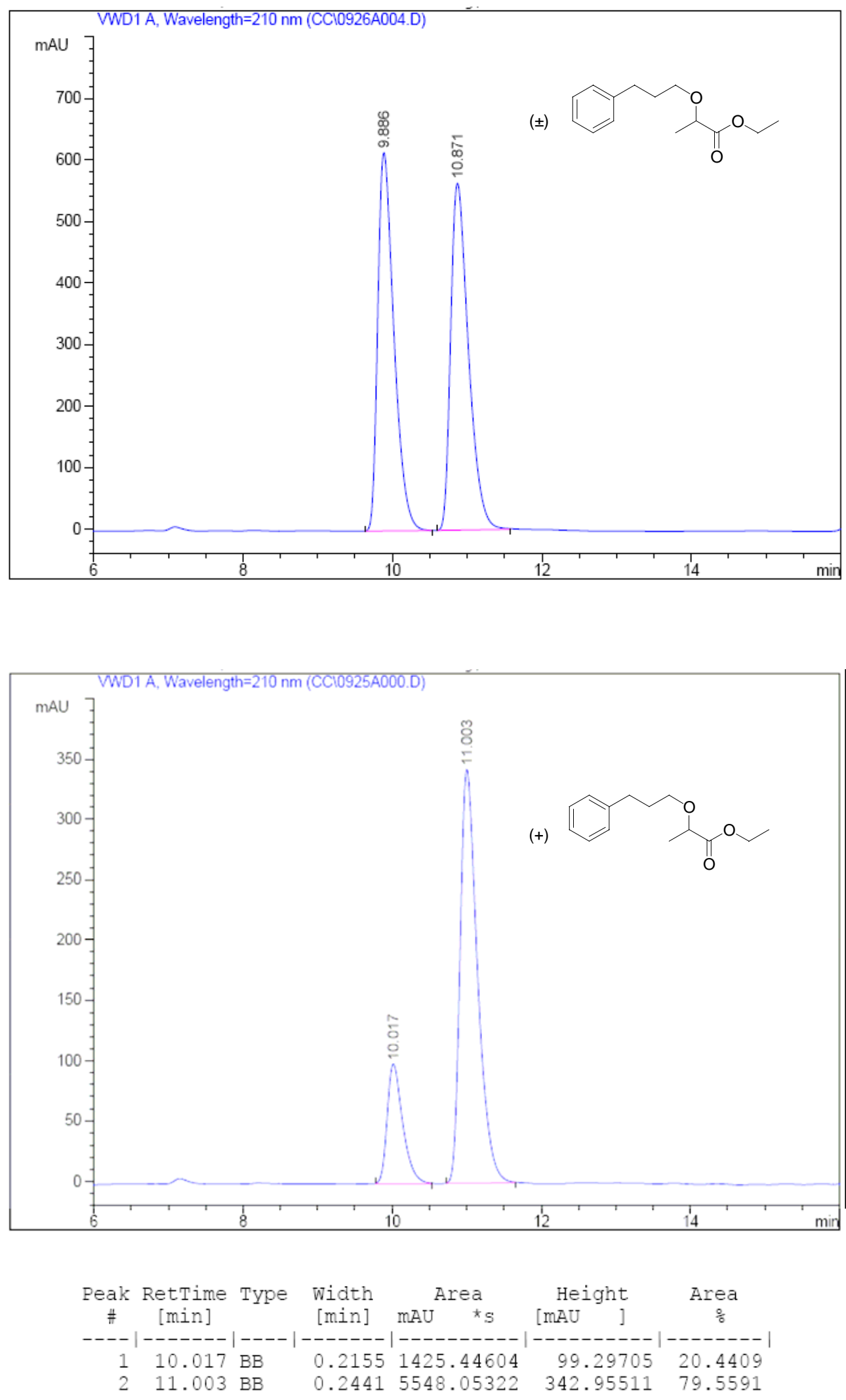


\section{(R)-(+)-Ethyl 2-(cinnamyloxy)propionate (4t)}
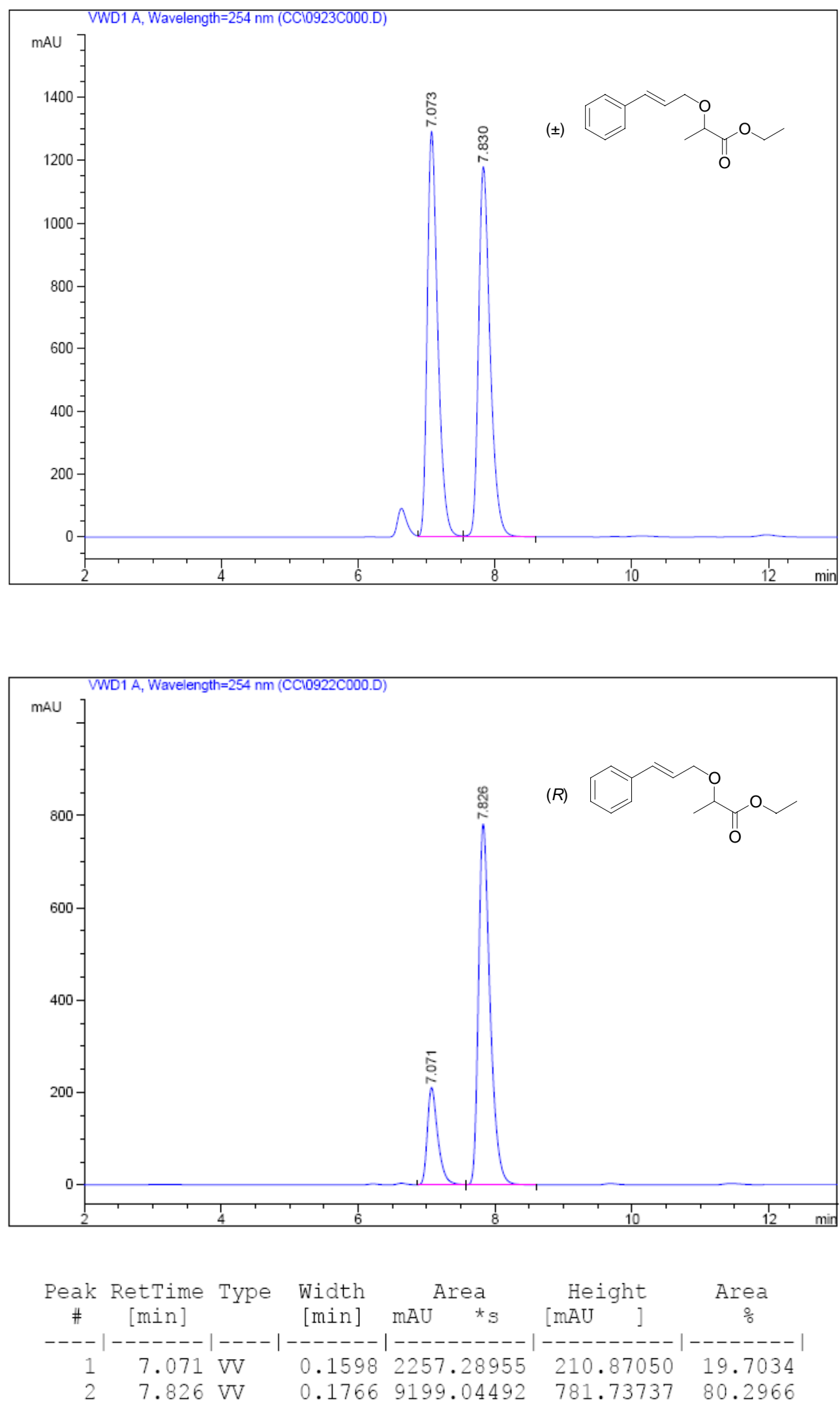
(R)-(-)-Methyl 2-phenoxy-2-phenylacetate (4u)
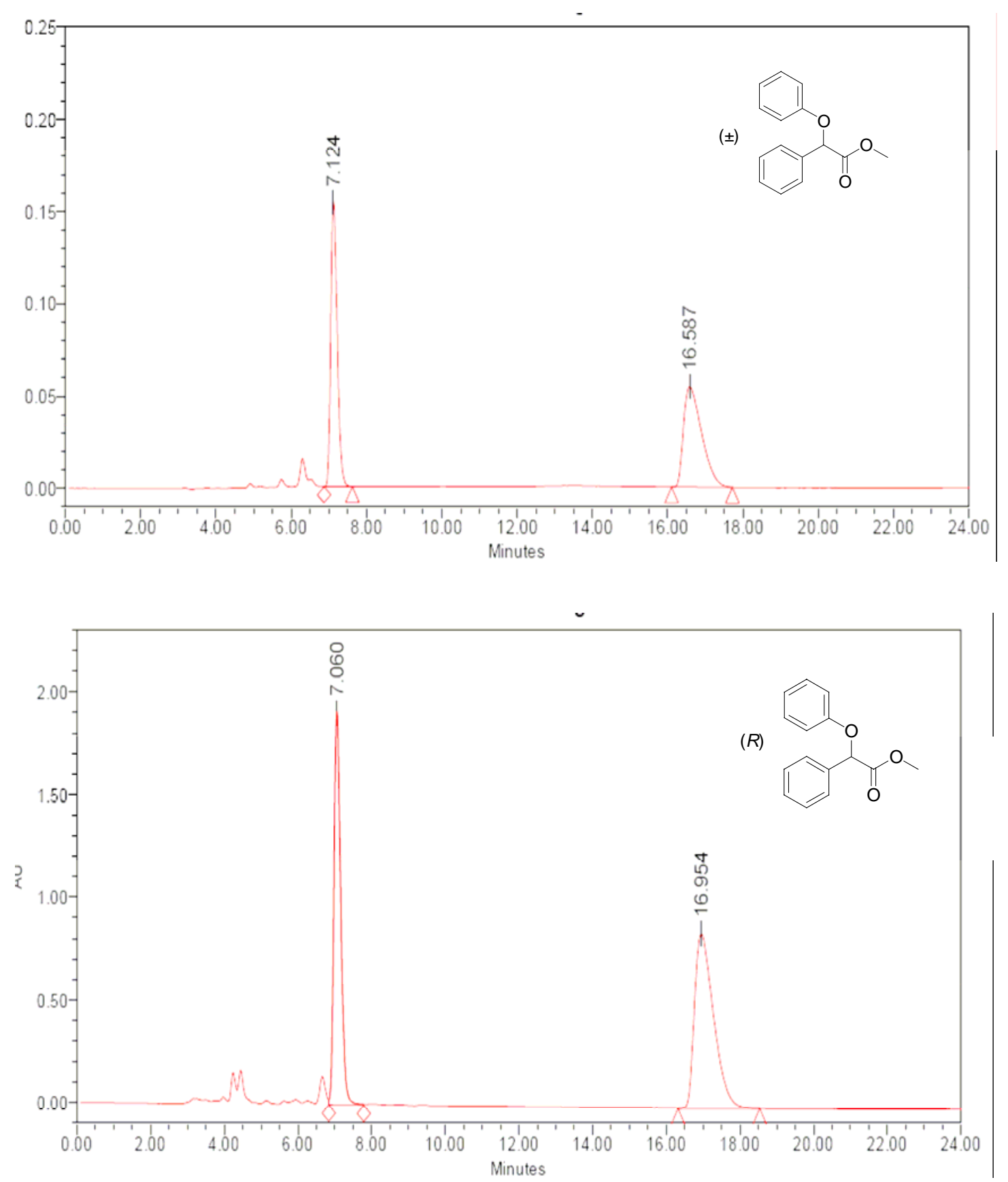

Peak Results

\begin{tabular}{|c|c|c|c|r|}
\hline & RT & Area & Height & $\%$ Area \\
\hline 1 & 7.060 & 24672976 & 1917123 & 44.78 \\
\hline 2 & 16.954 & 30419802 & 849414 & 55.22 \\
\hline
\end{tabular}


(R)-(+)-Methyl 2-phenoxypropionate (4v)
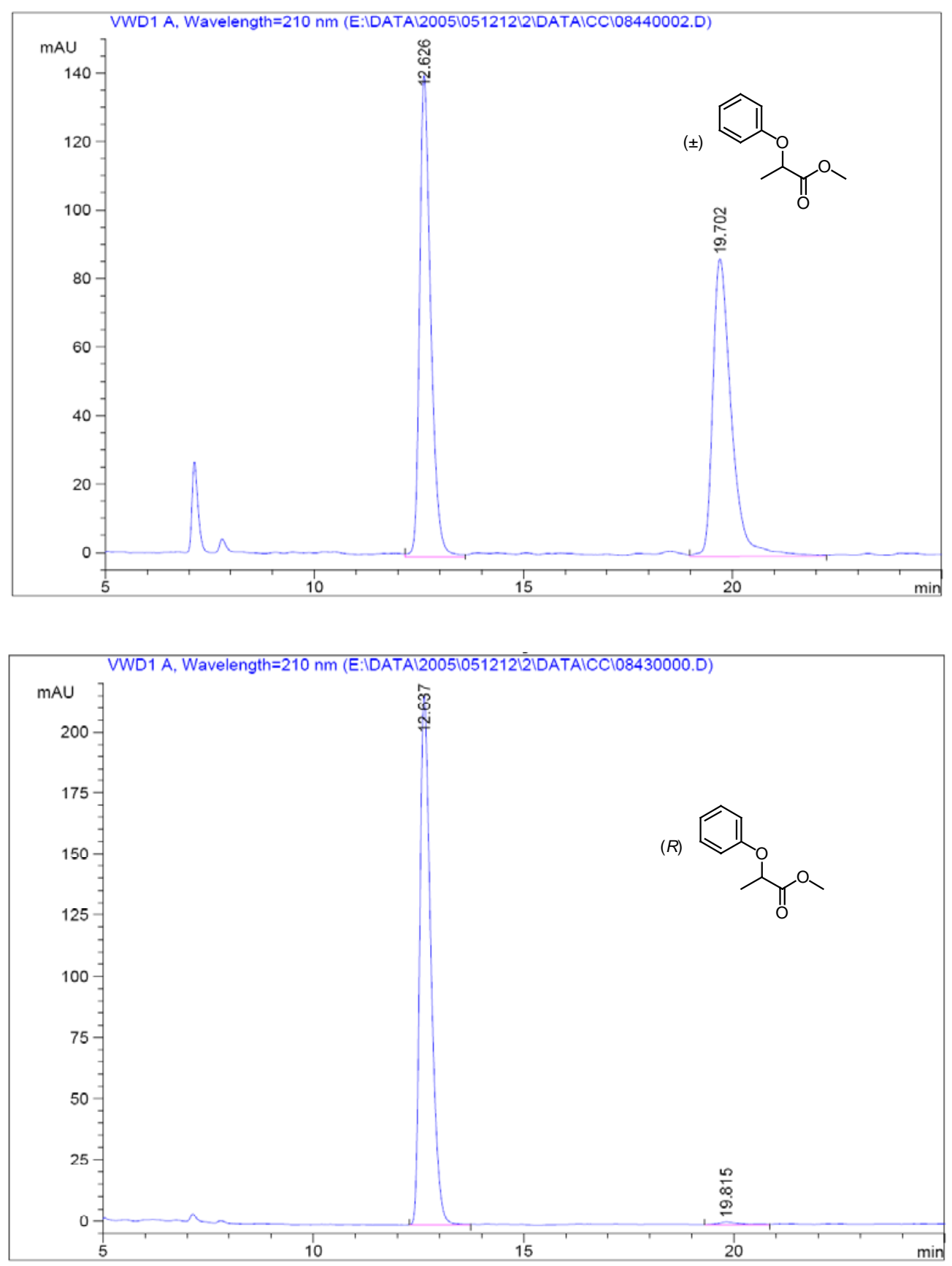

Signal 1: VWD1 A, Wavelength=210 nm

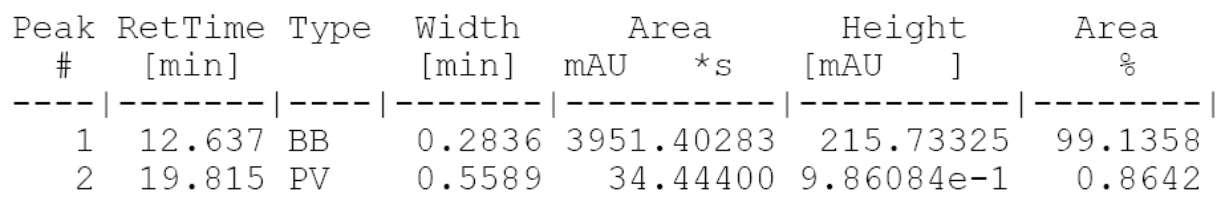

S40 
(+)-tert-butyl 2-phenoxypropionate (4w)
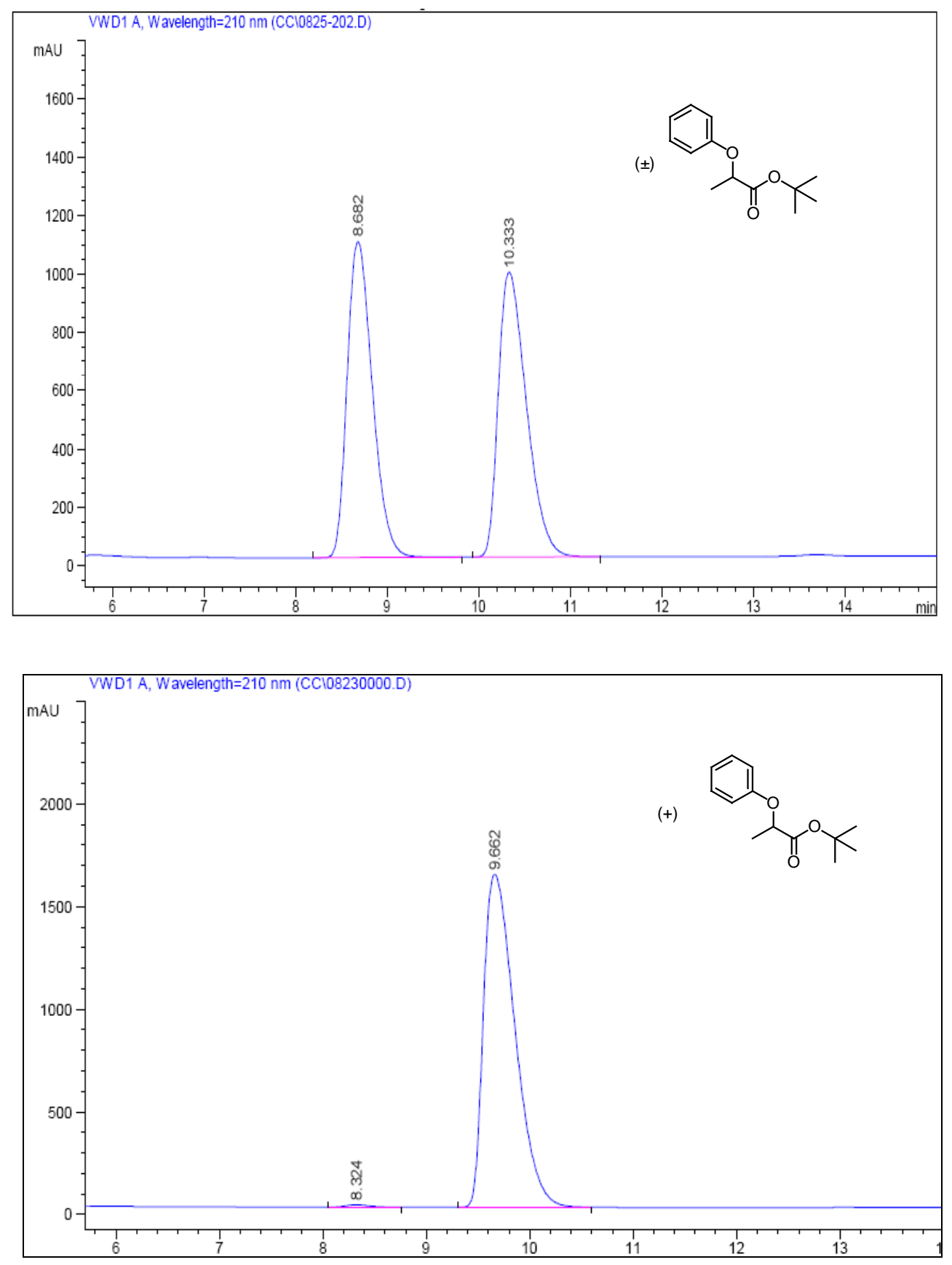

Signal 1: VWD1 A, Wavelength=210 nm

\begin{tabular}{|c|c|c|c|c|c|c|}
\hline Peak & RetTime & Type & width & Area & Height & Area \\
\hline \# & [min] & & [min] & mAU & {$[\mathrm{mAU}$} & $\frac{8}{8}$ \\
\hline 1 & 8.324 & $\mathrm{BB}$ & 0.2489 & 203.09727 & 12.42815 & 0.5896 \\
\hline 2 & 9.662 & BB & 0.3308 & $3.42412 e 4$ & 1621.80884 & 99.4104 \\
\hline
\end{tabular}

